\title{
Relativistic four-component linear damped response TDDFT for electronic absorption and circular dichroism calculations
}

Lukas Konecny, ${ }^{1,2, a)}$ Michal Repisky, ${ }^{1}$ Kenneth Ruud, ${ }^{1}$ and Stanislav Komorovsky ${ }^{3, b}$ )

${ }^{1)}$ Hylleraas Centre for Quantum Molecular Sciences, UiT The Arctic University of Norway, 9037, Tromsø, Norway

2) Department of Inorganic Chemistry, Faculty of Natural Sciences, Comenius University, Bratislava, Slovakia

3) Institute of Inorganic Chemistry, Slovak Academy of Sciences, Bratislava, Slovakia

(Dated: 3 November 2019)

We present a detailed theory, implementation, and a benchmark study of a linear damped response time-dependent density functional theory (TDDFT) based on the relativistic four-component (4c) Dirac-Kohn-Sham formalism using the restricted kinetic balance condition for the small-component basis and a non-collinear exchangecorrelation kernel. The damped response equations are solved by means of a multifrequency iterative subspace solver utilizing decomposition of the equations according to hermitian and time-reversal symmetry. This partitioning leads to robust convergence and the detailed algorithm of the solver for relativistic multicomponent wavefunctions is also presented. The solutions are then used to calculate the linear electricand magnetic-dipole responses of molecular systems to an electric perturbation, leading to frequency-dependent dipole polarizabilities, electronic absorption and circular dichroism (ECD), and optical rotatory dispersion (ORD) spectra. The methodology has been implemented in the relativistic spectroscopy DFT program ReSpect, and its performance assessed on a model series of dimethylchalcogeniranes, $\mathrm{C}_{4} \mathrm{H}_{8} \mathrm{X}(\mathrm{X}=\mathrm{O}$, $\mathrm{S}, \mathrm{Se}, \mathrm{Te}, \mathrm{Po}, \mathrm{Lv}$ ) and on larger transition metal complexes that have been studied experimentally, $\left[\mathrm{M}(\mathrm{phen})_{3}\right]^{3+}(\mathrm{M}=\mathrm{Fe}, \mathrm{Ru}, \mathrm{Os})$. These are the first $4 \mathrm{c}$ damped linear response TDDFT calculations of ECD and ORD presented in the literature.

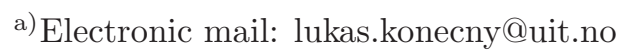

b)Electronic mail: stanislav.komorovsky@savba.sk
} 


\section{INTRODUCTION}

Time-dependent density functional theory (TDDFT) represents an extension of DFT to time-dependent context and has proven to be a successful method for calculating dynamical (frequency-dependent) molecular properties. ${ }^{1-4}$ However, calculations of these properties often demand proper inclusion of scalar and spin-orbit (SO) relativistic effects, particularly for systems containing heavy elements or when addressing core states. ${ }^{5}$ The "gold standard" of relativistic quantum chemistry is the four-component (4c) methodology that combines the one-electron Dirac Hamiltonian containing both scalar and SO effects non-perturbatively with instantaneous Coulomb interactions among the particles. Furthermore, approximate 2-component (2c) or scalar-relativistic (1c) Hamiltonians have been developed to reduce computational cost of relativistic calculations. ${ }^{6,7}$ TDDFT can be formulated at the relativistic level of theory and similarly to its non-relativistic case can be approached from three distinct directions.

The approach most commonly used in quantum chemistry is based on linear perturbation theory and takes advantage of the fact that poles of linear response functions correspond to excitation energies and can be calculated as eigenvalues from the eigenvalue response equation (often referred to as the Casida equation). ${ }^{8-10}$ Further molecular properties such as oscillator or rotatory strengths correspond to residues response functions and can be obtained from the eigenvectors ${ }^{11}$. The popularity of this approach for a wide range of systems is also due to efficient algorithms available for solving this equation. ${ }^{12-17}$ However, since eigenvalue calculations normally proceed from the lowest excitation energy and the computational cost increases with the number of eigenvalues, its applications in high-frequency spectral regions and regions with high density-of-states remain challenging and require a development of special techniques. ${ }^{15,16,18,19}$ Moreover, due to its perturbative nature, the response eigenvalue equation requires the evaluation of the derivatives of DFT exchangecorrelation potentials (so-called kernels) that must be formulated carefully, particularly in relativistic multi-component theories with spin-orbit coupling. ${ }^{17,20-22}$ Relativistic implementations of the response eigenvalue equations have been reported at the $4 \mathrm{c}$ level of theory for closed-shell ${ }^{20,23,24}$ as well as open-shell systems ${ }^{17}$, X2C level of theory ${ }^{22,25-27}$, scalar zerothorder regular approximation (ZORA) ${ }^{28,29}$ and spin-orbit $\mathrm{ZORA}^{30}$, and recently reviewed by Liu and Xiao. ${ }^{31}$ The scope of applications of relativistic linear response TDDFT with 
variational SO interaction includes absorption spectra in valence ${ }^{32-34}$ and X-ray regions ${ }^{19}$, excited-state zero-field splittings, ${ }^{27}$ and phosphorescence lifetimes ${ }^{35-37}$.

An alternative perturbation theory-based strategy is to directly determine the response of a molecular system to an external field of a particular frequency by solving either the standard response equation or the damped response equation (also known in literature as the complex polarization propagator approach).$^{38-40}$ The latter includes an imaginary damping parameter to prevent divergencies at near-resonant frequencies and to allow treatment absorption processes, a limitation of the former. The advantage of damped response theory over the eigenvalue response approach lies in its straightforward applicability in high-frequency or high density-of-states spectral regions, since for many chemical applications a spectral function rather than a plethora of excitation energies is desired. Moreover, the possibility to solve the response equation with purely imaginary frequencies allows $\mathrm{C}_{6}$ dispersion coefficients to be efficiently calculated ${ }^{41}$. The computational cost is similar to the eigenvalue response equation and DFT kernels are required also in this case. In the context of relativistic theories with variational SO interaction, the methodology has been developed in the spin-orbit ZORA $^{42}$, and $4 \mathrm{c}^{43,44}$ framework and applied to the calculation of frequency-dependent dipole polarizabilities ${ }^{45}$, electronic absorption spectra in valence and X-ray regions ${ }^{46,47}$, and electric dipole dispersion interaction coefficients ${ }^{48}$. An extensive list of review texts on eigenvalue and damped response theory can be found in Table 1 in Ref. 18.

Finally, so-called real-time TDDFT (RT-TDDFT) solves the equation of motion nonperturbatively in the time domain, in contrast to the previous response-theory, frequencydomain approaches. ${ }^{49-51}$ RT-TDDFT allows molecules in strong time-dependent external fields of arbitrary shape to be described, and can address near-resonant frequencies and various spectral regions in a single run. It also does not require the evaluation of response kernels. However, the methodology suffers from an increased computational cost for many chemical applications where it suffices to describe molecular response properties to the first few lowest orders for a narrow frequency range. Implementations have been reported at the $4 \mathrm{c}$ level of theory and used to calculate electron absorption spectra in valence ${ }^{52}$ and $\mathrm{X}-$ ray $^{53}$ regions and circular dichroism spectra ${ }^{54}$, as well as at the $2 \mathrm{c} \mathrm{X} 2 \mathrm{C}$ level of theory and applied to electron absorption spectra in the valence $e^{55}$ and $\mathrm{X}-$ ray $^{56}$ regions, and non-linear optical properties $^{57}$.

This work focuses on the linear damped response theory, the derivation of its work- 
ing equation based on time-dependent perturbation theory formulated for general multicomponent spinor cases $(2 \mathrm{c}, 4 \mathrm{c})$, and the description of a multi-frequency iterative subspace solver accounting for hermicity and time-reversal symmetry. The specific form of the noncollinear kernel and subspace solver restrict the applicability of the presented methodology to systems with a non-degenerate ground state (closed-shell singlet configuration). The methodology is applied to model series of dimethylchalcogeniranes, $\mathrm{C}_{4} \mathrm{H}_{8} \mathrm{X}(\mathrm{X}=\mathrm{O}, \mathrm{S}, \mathrm{Se}$, $\mathrm{Te}, \mathrm{Po}, \mathrm{Lv})$ and to larger transition metal complexes of experimental interest, $\left[\mathrm{M}(\mathrm{phen})_{3}\right]^{3+}$ $(\mathrm{M}=\mathrm{Fe}, \mathrm{Ru}, \mathrm{Os})$ calculating their polarizabilities, electron absorption, electronic circular dichroism (ECD) and optical rotatory dispersion (ORD) spectra, the latter two constituting the first 4c relativistic linear damped response ECD and ORD spectra presented in the literature. Together with developments described in earlier works ${ }^{17,52}$ it endows our program package ReSpect ${ }^{58}$ with all three TDDFT approaches outlined above. Therefore, the users may choose the most suitable method for the chemical problem at hand within a single program.

The structure of this article is as follows. It starts with a detailed theory derivation of the damped response equation in Section II A, followed in Section IIB, by the details of the relativistic formalism including a non-collinear exchange-correlation kernel. The theoretical section ends with the discussion of the connection between the calculated response function and experimentally observable molecular properties. In Section III, the technical details of the iterative subspace algorithm for the damped response equation adjusted for the $4 \mathrm{c}$ framework by the consideration of hermicity and time reversal symmetry are laid out. Section IV presents the computational details, and finally, Section V contains the results of pilot applications. The paper ends with concluding remarks and perspectives for further development.

\section{THEORY}

In this work we employ the following conventions: subscripts $i, j$ denote occupied, $a$, $b$ virtual and $p, q, s, t$ general molecular orbitals; subscripts $\mu, \nu$ denote basis functions; and subscripts $u$ and $v$ denote Cartesian components. Einstein's summation convention is assumed. Unless specified otherwise, formulas are written in atomic units. 


\section{A. Damped response equation}

The aim of damped response theory is to describe a molecular system under the influence of an external field with relaxation and finite lifetimes of excited states included. A common formulation is based on density matrices that evolve in time according to the Liouville-von Neumann ( $\mathrm{LvN})$ equation $^{59}$. In TDDFT, the LvN equation for the reduced one-electron density matrix D represented in the basis of static Kohn-Sham (KS) molecular orbitals (MOs) reads

$$
i \frac{\partial}{\partial t} D_{p q}(t)=\left[F^{\prime}(t), D(t)\right]_{p q}-i \gamma_{p q}\left(D_{p q}(t)-D_{p q}^{\mathrm{eq}}\right),
$$

where $\mathbf{F}^{\prime}(t)$ is the Fock matrix characterizing the molecular system of interest and $\gamma_{p q}$ is the damping factor describing the rate of relaxation of the density matrix element $D_{p q}(t)$ towards its equilibrium value $D_{p q}^{\mathrm{eq}}$. Since in this work we neglect thermal electronic excitations, the equilibrium corresponds to the ground state, i.e. $D_{p q}^{\mathrm{eq}}=\delta_{p i} \delta_{q i}$, with $\delta_{p i}$ being the Kronecker delta and index $i$ spanning over occupied MOs. The damping matrix $\gamma$ has zero occupiedoccupied block and its non-zero parts describe inverse lifetimes of excited states, which leads to finite-width peaks in the spectra. The one-electron reduced density matrix elements in the basis of static KS MOs $\varphi(r)$ are expressed as

$$
D_{p q}(t)=\left\langle\boldsymbol{\varphi}_{p}(\boldsymbol{r}) \mid \boldsymbol{\varphi}_{i}(\boldsymbol{r}, t)\right\rangle\left\langle\boldsymbol{\varphi}_{i}(\boldsymbol{r}, t) \mid \boldsymbol{\varphi}_{q}(\boldsymbol{r})\right\rangle
$$

where $\boldsymbol{r}$ is the electronic coordinate and the static orbitals are the solutions of the timeindependent KS equation. Since the main focus of this work is on relativistic theory, the bold font used for $\varphi$ refers to their multicomponent spinor structure (2c or 4c) and $\langle\cdot \mid \cdot\rangle$ in Eq. (2) involves both the spatial integration and trace over the spinor elements. The Fock matrix in Eq. (1) is composed of a field-free part $\mathbf{F}$ and a perturbation $\mathbf{V}_{\omega}^{(1)}$ describing the coupling of the system to a time-dependent external field of frequency $\omega$ and a small amplitude $\lambda$

$$
\mathbf{F}^{\prime}(t) \equiv \mathbf{F}[\mathbf{D}(t)]+\lambda \mathbf{V}_{\omega}^{(1)}(t)
$$

In addition to explicit time dependence of $\mathbf{F}^{\prime}(t)$ via $\mathbf{V}_{\omega}^{(1)}(t)$, the field-free part is implicitly time dependent via the density matrix. Within response theory, the perturbation operator is customarily chosen to have the form ${ }^{59}$

$$
\mathbf{V}_{\omega}^{(1)}(t) \equiv \mathbf{P} e^{-i \omega t+\eta t}+\mathbf{P}^{\dagger} e^{i \omega t+\eta t}
$$


where $\mathbf{P}$ is matrix representation of the spatial part of the external field operator. Even though $\mathbf{P}$ in Eq. (4) is general, in the the rest of this paper we will assume that it is hermitian. Specifically, for the applications presented, we assume interaction of a system with an external electric field, which leads within an electric dipole approximation leading to $\hat{P}=-\hat{\mu}$, where $\hat{\mu}$ is the electric dipole moment operator. The temporal component of $\mathbf{V}_{\omega}^{(1)}(t)$ corresponds to a harmonic field of frequency $\omega$ with a field-switching factor $\eta$. The term $\exp [\eta t]$ describes the field being slowly switched on and $\eta$ being small ensures a smooth application of the perturbation. In the end, the limit $\eta \rightarrow 0$ will be considered. While the perturbation $\mathbf{V}_{\omega}^{(1)}(t)$ is assumed to consist of a single frequency component, generalization to more frequencies is straightforward.

While a direct propagation of the TDDFT equation of motion in time results in RT$\mathrm{TDDFT}^{51}$, response theory seeks for the solution of this equation via a perturbation expansion in powers of $\lambda$

$$
D_{p q}(t)=D_{p q}^{(0)}(t)+\lambda D_{p q}^{(1)}(t)+\frac{1}{2} \lambda^{2} D_{p q}^{(2)}(t)+\ldots,
$$

where the expansion point in Eq. (5) is provided by the solution of Eq. (1) without any external field, i.e. the ground-state density matrix, $D_{p q}^{(0)}(t)=D_{p q}^{\mathrm{eq}}=\delta_{p i} \delta_{q i}$. If the timedependent occupied MOs are parametrized via static KS MOs and complex time-dependent expansion coefficients $d_{p i}(t)$

$$
\boldsymbol{\varphi}_{i}(\boldsymbol{r}, t) \equiv \boldsymbol{\varphi}_{p}(\boldsymbol{r}) d_{p i}(t)
$$

the first-order correction to the time-dependent density matrix can be expressed via the first-order expansion coefficients $d_{p i}^{(1)}(t)$ as

$$
D_{p q}^{(1)}(t)=d_{p i}^{(1)}(t) \delta_{q i}+\delta_{p i} d_{q i}^{(1) *}(t)
$$

The normalization condition $\left\langle\boldsymbol{\varphi}_{i}(t) \mid \boldsymbol{\varphi}_{j}(t)\right\rangle=\delta_{i j}$, then gives in the first order

$$
d_{i j}^{(1)}(t)+d_{j i}^{(1) *}(t)=0
$$

that together with the Kronecker deltas in Eq. (7) leads to the following form of the density matrix

$$
\begin{aligned}
& D_{i j}^{(1)}(t)=D_{a b}^{(1)}(t)=0 \\
& D_{a i}^{(1)}(t)=D_{i a}^{(1) *}(t)=d_{a i}^{(1)}(t) .
\end{aligned}
$$


Therefore, it is sufficient to consider only the virtual-occupied block of the density matrix, i.e. the coefficients $d_{a i}^{(1)}(t)$. The final differential equation for the first-order perturbation coefficients $\mathbf{d}^{(1)}$ following from the Liouville-von Neumann equation to first order thus reads

$$
\begin{aligned}
i \frac{d}{d t} d_{a i}^{(1)}(t) & =F_{a i}^{(1)}\left[\mathbf{d}^{(1)}(t)\right]+\omega_{a i} d_{a i}^{(1)}-i \gamma_{a i} d_{a i}^{(1)} \\
& +P_{a i} e^{-i \omega t+\eta t}+P_{a i} e^{i \omega t+\eta t}
\end{aligned}
$$

where no summation is assumed in the second and third terms on the right-hand side and $\omega_{a i}=\epsilon_{a}-\epsilon_{i}$ with $\epsilon_{p}$ being the orbital energy of the $p$-th molecular orbital. The field-free part of the Fock operator in Eq. (3) is commonly combined with the term $\omega_{a i} d_{a i}^{(1)}$ and leads to the contribution in the form

$$
F_{a i}^{(1)}\left[\mathbf{d}^{(1)}\right]+\omega_{a i} d_{a i}^{(1)}=A_{a i, b j} d_{b j}^{(1)}+B_{a i, b j} d_{b j}^{(1) *}
$$

where the matrices $\mathbf{A}$ and $\mathbf{B}$ are defined as

$$
\begin{aligned}
A_{a i, b j} & \equiv \delta_{i j} \delta_{a b} \omega_{a i}+[a i \mid j b]-[a b \mid j i]+K_{a i, b j}^{\mathrm{XC}} \\
B_{a i, b j} & \equiv[a i \mid b j]-[a j \mid b i]+K_{a i, b j}^{\mathrm{XC}} .
\end{aligned}
$$

The matrix $\mathbf{K}^{\mathrm{XC}}$ is the exchange-correlation DFT kernel, and $[p q \mid s t]$ denote the four-centre electron repulsion integrals in MO basis. Detailed form of these terms in a 4c relativistic theory is the subject of section IIB. Since $\mathbf{F}\left[\mathbf{d}^{(1)}\right]$ contains both coefficients $\mathbf{d}^{(1)}$ as well as their complex conjugates $\mathbf{d}^{(1) *}$, Eq. (10) has to be solved as a coupled system of equations

$$
\begin{aligned}
i \frac{d}{d t}\left(\begin{array}{c}
\mathbf{d}^{(1)}(t) \\
\mathbf{d}^{(1) *}(t)
\end{array}\right) & =\left(\begin{array}{cc}
\mathbf{A}-i \boldsymbol{\gamma} & \mathbf{B} \\
-\mathbf{B}^{*} & -\mathbf{A}^{*}-i \boldsymbol{\gamma}
\end{array}\right)\left(\begin{array}{c}
\mathbf{d}^{(1)}(t) \\
\mathbf{d}^{(1) *}(t)
\end{array}\right) \\
& +\left(\begin{array}{c}
\mathbf{P} e^{-i \omega t+\eta t}+\mathbf{P} e^{i \omega t+\eta t} \\
-\mathbf{P}^{*} e^{i \omega t+\eta t}-\mathbf{P}^{*} e^{-i \omega t+\eta t}
\end{array}\right),
\end{aligned}
$$

where $\mathbf{d}^{(1)}$ and $\mathbf{P}$ are complex matrices of size $N_{\mathrm{v}} \times N_{\mathrm{o}}$ with $N_{\mathrm{v}}$ and $N_{\mathrm{o}}$ referring to the number of virtual and occupied MOs, respectively.

The differential equation in Eq. (13) can be turned into an algebraic form by the method of undetermined coefficients, substituting

$$
\mathbf{d}^{(1)}(t) \equiv \mathbf{X} e^{-i \omega t+\eta t}+\mathbf{Y}^{*} e^{i \omega t+\eta t}
$$


where $\mathbf{X}$ and $\mathbf{Y}$ are complex matrices of time-independent undetermined coefficients. After substituting Eq. (14) into Eq. (13) and collecting terms proportional to $e^{-i \omega t+\eta t}$ one arrives at the final linear damped response equation

$$
\left[\left(\begin{array}{cc}
\mathbf{A} & \mathbf{B} \\
\mathbf{B}^{*} & \mathbf{A}^{*}
\end{array}\right)-(\omega+i \gamma)\left(\begin{array}{cc}
\mathbf{1} & \mathbf{0} \\
\mathbf{0} & -\mathbf{1}
\end{array}\right)\right]\left(\begin{array}{l}
\mathbf{X} \\
\mathbf{Y}
\end{array}\right)=-\left(\begin{array}{c}
\mathbf{P} \\
\mathbf{P}^{*}
\end{array}\right),
$$

where the common damping factor $\gamma \equiv \gamma_{a i}$ was used for all virtual-occupied pairs, and the limit $\eta \rightarrow 0$ was considered. The equation proportional to $e^{i \omega t+\eta t}$ is just a complex conjugate of Eq. (15) with identical solution. Section III discusses the iterative subspace method for the solution of Eq. (15) as implemented in our program.

Another notation for the damped response equation commonly found in literature results from the Ehrenfest theorem-based derivation ${ }^{60}$

$$
\left[\mathbf{E}^{[2]}-(\omega+i \gamma) \mathbf{S}^{[2]}\right] \mathbf{Z}=\mathbf{G},
$$

whose objects correspond term-by-term to Eq. (15). The right-hand side $\mathbf{G}$ is the property gradient, $\mathbf{Z}$ is the response vector containing matrices $\mathbf{X}$ and $\mathbf{Y}, \mathbf{S}^{[2]}$ is the metric matrix, and $\mathbf{E}^{[2]}$ is the generalized Hessian. Without the damping factor the equation is referred to as the standard response equation ${ }^{61}$

$$
\left[\mathbf{E}^{[2]}-\omega \mathbf{S}^{[2]}\right] \mathbf{Z}=\mathbf{G},
$$

while the equation without the property gradient is the well-known Casida equation ${ }^{8}$

$$
\mathbf{E}^{[2]} \mathbf{Z}=\omega_{f} \mathbf{S}^{[2]} \mathbf{Z}
$$

which is the eigenvalue equation for the excitation energies $\omega_{f}$. Note also that TDDFT is closely related to another popular method, the random phase approximation or timedependent Hartree-Fock method, which amounts to the neglect of the exchange-correlation kernel in $\mathrm{TDDFT}^{62}$. In addition, the response eigenvalue equation is often decoupled by neglecting the $\mathbf{B}$ term in $\mathbf{E}^{[2]}$, leading to the Tamm-Dancoff approximation in TDDFT ${ }^{63}$ where the dimensions of all matrices and vectors are reduced to half.

\section{B. Damped response equation in $4 \mathrm{c}$ relativistic theory}

The derivation in Section II A proceeded in a general fashion, i.e. working equations were derived and formulated in a way applicable to both Kohn-Sham DFT or Hatree-Fock 
theory, regardless of the level of relativistic theory used (1c, 2c, 4c). In this section, we delve into the details of the terms appearing in Eqs. (11) and (12) that are specific for 4c theory.

Here, we assume that the 4c canonical MOs $\boldsymbol{\varphi}_{i}(\boldsymbol{r})$ and corresponding one-electron energies $\epsilon_{i}$ were obtained by solving the 4c Dirac-Kohn-Sham equation (see for example Refs. 64 and 65) in the restricted kinetic balance (RKB) basis

$$
\mathbf{X}_{\mu}(\boldsymbol{r}) \equiv\left(\begin{array}{cc}
\boldsymbol{\sigma}_{0} & \mathbf{0} \\
\mathbf{0} & \frac{1}{2 c}(\boldsymbol{\sigma} \cdot \boldsymbol{p})
\end{array}\right) \chi_{\mu}(\boldsymbol{r}),
$$

where $\boldsymbol{\sigma}_{0}$ is a $2 \times 2$ unit matrix, $\boldsymbol{\sigma}$ is a vector composed of Pauli matrices, and the functions $\chi_{\mu}(\boldsymbol{r})$ are elements of a real scalar basis set, in our implementation chosen as Gaussian-type orbitals (GTO). The MOs are expanded in the RKB basis as

$$
\boldsymbol{\varphi}_{p}(\boldsymbol{r}) \equiv \mathbf{X}_{\mu}(\boldsymbol{r}) \mathbf{C}_{\mu p}
$$

and the 4c overlap distribution functions in the MO basis $\boldsymbol{\varphi}_{p}(\boldsymbol{r})$ are defined as

$$
\Omega_{p q}^{m}(\boldsymbol{r}) \equiv \boldsymbol{\varphi}_{p}^{\dagger}(\boldsymbol{r}) \boldsymbol{\Sigma}_{m} \boldsymbol{\varphi}_{q}(\boldsymbol{r})=\left(\mathbf{X}_{\mu} \mathbf{C}_{\mu p}\right)^{\dagger} \boldsymbol{\Sigma}_{m} \mathbf{X}_{\nu} \mathbf{C}_{\nu q}
$$

with the spin operator

$$
\boldsymbol{\Sigma}_{m} \equiv\left(\begin{array}{cc}
\boldsymbol{\sigma}_{m} & \mathbf{0} \\
\mathbf{0} & \boldsymbol{\sigma}_{m}
\end{array}\right), \quad m \in 0 \ldots 3 .
$$

The 4-centre electron repulsion integrals in Eq. (12) then take the form

$$
[p q \mid s t] \equiv \iint \Omega_{p q}^{0}\left(\boldsymbol{r}_{1}\right) \frac{1}{r_{12}} \Omega_{s t}^{0}\left(\boldsymbol{r}_{2}\right) \mathrm{dV}_{1} \mathrm{dV} \mathrm{V}_{2}
$$

Efficient evaluation of these integrals in the relativistic regime can be facilitated by using quaternion algebra as was previously discussed for 2c and 4c RT-TDDFT ${ }^{54}$ and relativistic periodic solid state calculations ${ }^{66}$.

The present work considers only molecular systems with non-degenerate ground states, for which the electron spin density and its gradients are zero at every point of space, and therefore do not enter the evaluation of the exchange-correlation (XC) potential used to obtain the canonical MOs. As a result, the XC potential in the adiabatic approximation has the form of Eq. (20) in Ref. 65. However, the first derivatives of the XC potential with respect to the electron spin density and its gradients are non-zero and result in the following 
non-collinear XC kernel ${ }^{17}$

$$
\begin{aligned}
& K_{p q s t}^{\mathrm{XC}}= \\
& \int\left\{\left(k^{n n} \Omega_{s t}^{0}+k^{n g_{n n}} 2 \Gamma_{s t}^{0}\right) \Omega_{p q}^{0}\right. \\
& +\left(k^{n g_{n n}} \Omega_{s t}^{0}+k^{g_{n n} g_{n n}} 2 \Gamma_{s t}^{0}\right) 2 \Gamma_{p q}^{0} \\
& +\left(k^{s g_{n s}} \Omega_{s t}^{k}+k^{g_{n s} g_{n s}} \Gamma_{s t}^{k}\right) \Gamma_{p q}^{k} \\
& +\left(k^{s s} \Omega_{s t}^{k}+k^{s g_{n s}} \Gamma_{s t}^{k}\right) \Omega_{p q}^{k} \\
& \left.+v^{g_{n n}} 2 \vec{\nabla} \Omega_{s t}^{0} \cdot \vec{\nabla} \Omega_{p q}^{0}+v^{g_{s s}} 2 \vec{\nabla} \Omega_{s t}^{k} \cdot \vec{\nabla} \Omega_{p q}^{k}\right\} \mathrm{dV},
\end{aligned}
$$

with auxiliary variables

$$
\begin{aligned}
\Gamma_{s t}^{m} & \equiv \vec{\nabla} \rho_{0} \cdot \vec{\nabla} \Omega_{s t}^{m}, \\
v^{w} & \equiv \frac{\partial \varepsilon^{\mathrm{XC}}}{\partial u}, \quad w=g_{n n}, g_{s s}, \\
k^{w f} & \equiv \frac{\partial \varepsilon^{\mathrm{xc}}}{\partial u \partial f}, \quad w, f=n, s, g_{n n}, g_{s s}, g_{n s},
\end{aligned}
$$

defined through the exchange-correlation energy density $\varepsilon^{\mathrm{xc}}$, the electron density $\rho_{0}=\Omega_{i i}^{0}$, spin densities $\rho_{m}=\Omega_{i i}^{m}, m=1 \ldots 3, n=\rho_{0}, s=0$, and variables

$$
\begin{aligned}
g_{n n} & =\vec{\nabla} \rho_{0} \cdot \vec{\nabla} \rho_{0}, \\
g_{n s} & =0, \\
g_{s s} & =\vec{\nabla} \rho_{k} \cdot \vec{\nabla} \rho_{k}, \quad k=1 \ldots 3 .
\end{aligned}
$$

The potential and kernel functions in Eqs. (25b) and (25c) are defined in the non-collinear fashion, where the non-relativistic collinear variables $\rho_{z}, \vec{\nabla} \rho_{0} \cdot \vec{\nabla} \rho_{z}$, and $\vec{\nabla} \rho_{z} \cdot \vec{\nabla} \rho_{z}$ are replaced by their non-collinear counterparts $s, g_{n s}$, and $g_{s s}$, respectively. The non-collinear kernel in Eq. (24) builds on the previous work for local functionals ${ }^{24,29,30,67-69}$, includes the extension towards GGA functionals by Bast et al. ${ }^{20}$, and involves our adaptation for the use of RKB instead of the unrestricted kinetic balance basis considered in the original work ${ }^{20}$.

\section{Linear response functions}

The aim of damped response theory is the calculation of frequency-dependent molecular properties. ${ }^{59}$ To obtain the final formulas, let us first consider a time-dependent observable 
$R(t)$ as a response to a general perturbation with a small amplitude $\lambda$ and time dependence described by a function $F(t)$. The response can be expanded in the Volterra series in the powers of the external field

$$
\begin{aligned}
R(t) & =R^{(0)}+\lambda \int_{-\infty}^{\infty} \chi^{(1)}\left(t-t^{\prime}\right) F\left(t^{\prime}\right) d t^{\prime} \\
& +\frac{\lambda^{2}}{2} \iint_{-\infty}^{\infty} \chi^{(2)}\left(t-t^{\prime}, t-t^{\prime \prime}\right) F\left(t^{\prime}\right) F\left(t^{\prime \prime}\right) d t^{\prime} d t^{\prime \prime}+\ldots
\end{aligned}
$$

where the real functions $\chi^{(n)}$ are $n$-th order susceptibilities. Considering $F(t)$ to be a harmonic function with damping according to Eq. (4), the first-order contribution becomes

$$
R^{(1)}(t)=\int_{-\infty}^{\infty} \chi^{(1)}\left(t-t^{\prime}\right)\left(e^{-i \omega t^{\prime}+\eta t^{\prime}}+e^{+i \omega t^{\prime}+\eta t^{\prime}}\right) d t^{\prime}
$$

Note that the perturbation can in general vary in space which would result in more convolutions with integrations over the spatial variables. However, since in this work we consider only local interactions with external fields (the dipole approximation), we omit the spatial dependence. Moreover, the response and perturbation are in general of vector nature, making the susceptibilities tensor quantities. By definition, the first-order susceptibility obeys $\chi^{(1)}\left(t-t^{\prime}\right)=0$, for $t^{\prime}>t$ to preserve causality and to allow us to formulate its frequency-domain component by means of a Laplace transform. The frequency-dependent susceptibility

$$
\chi^{(1)}(\omega) \equiv \int_{-\infty}^{\infty} \chi^{(1)}(\tau) e^{+i \omega \tau-\eta \tau} d \tau
$$

can be recognized in Eq. (28), allowing us to write the first-order correction to $R$ in the form

$$
R^{(1)}(t)=\left[\chi^{(1)}(\omega) e^{-i \omega t+\eta t}+\chi^{(1) *}(\omega) e^{+i \omega t+\eta t}\right]
$$

where the symmetry $\chi^{(1)}(-\omega)=\chi^{(1) *}(\omega)$ of the, in general complex, frequency-dependent linear susceptibility, was considered.

A connection between $\chi^{(1)}(\omega)$ in Eq. (30) and solutions of the response equation in Eq. (15) can be established by considering $R(t)$ as an expectation value

$$
\begin{aligned}
R(t) & =\operatorname{Tr}[\mathbf{R D}(t)] \\
& =\operatorname{Tr}\left[\mathbf{R} \mathbf{D}^{(0)}(t)\right]+\lambda \operatorname{Tr}\left[\mathbf{R} \mathbf{D}^{(1)}(t)\right]+\ldots,
\end{aligned}
$$

where $\mathbf{R}$ is the matrix representation of the one-electron property operator associated with $R(t)$. In Eq. (31b) the first term on the right-hand side corresponds to the zeroth-order 
(static) contribution while the following two terms represent the first-order correction $\left(R^{(1)}\right)$. After expanding $\mathbf{D}(t)$ according to Eq. (5), $R^{(1)}$ reads

$$
R^{(1)}(t)=\left(d_{a i}^{(1)}\right)^{*} R_{a i}+d_{a i}^{(1)} R_{i a}
$$

which by utilizing ansatz in Eq. (14) can be written as

$$
\begin{aligned}
R^{(1)}(t) & =\left(X_{a i} R_{i a}+Y_{a i} R_{a i}\right) e^{-i \omega t+\eta t} \\
& +\left(X_{a i}^{*} R_{a i}+Y_{a i}^{*} R_{i a}\right) e^{+i \omega t+\eta t}
\end{aligned}
$$

The final formula used in practical calculations of the frequency-dependent linear response function can be obtained by comparing Eq. (33) with Eq. (30)

$$
\chi^{(1)}(\omega)=X_{a i} R_{i a}+Y_{a i} R_{a i} .
$$

Here, $\mathbf{X}=\mathbf{X}(\omega, \gamma)$ and $\mathbf{Y}=\mathbf{X}(\omega, \gamma)$ are solutions of the linear damped response equation, Eq. (15). The notation $\langle\langle R ; P\rangle\rangle_{\omega}$ for a linear response function describing a response $R$ to a perturbation $P$ is often used instead of the first-order susceptibility. However, when defining $\langle\langle R ; P\rangle\rangle_{\omega}$, only the bare perturbation operator is used, resulting in a need for a prefactor when transforming it into $\chi^{(1)}(\omega)$.

The frequency-dependent susceptibility is real or complex, depending on whether the solutions of standard $(\gamma=0)$ or damped response equation (Eqs. (17) and (16), respectively) were used in Eq. (34). In the case of complex susceptibilities, the real and imaginary parts describe dispersion and absorption, respectively. The calculation of spectra amounts to solving the linear response equation for a number of frequencies in the spectral range of interest. This is contrasted with the RT-TDDFT approach where the system has to be propagated for a sufficiently long time interval in order to yield the desired spectral resolution with a small time step (to ensure the stability of the propagation), typically resulting in a much larger spectral range than necessary and, in turn, requiring a larger number of expensive computational tasks.

The spectrum obtained from damped response theory can be analyzed in terms of transitions between ground-state MOs, by considering individual virtual-occupied orbital-pair contributions $\chi_{a i}^{(1)}(\omega)$ (Eq. (34) without the summation over repeated indices). This is analogous to the dipole-weighted transition analysis introduced earlier in the context of RT$\operatorname{TDDFT}^{52,53}$. 


\section{Electron absorption spectroscopy (EAS) and frequency-dependent linear electric dipole polarizability}

The first complex response property considered in this work is the complex polarizability tensor that connects the induced electric dipole moment to an applied electric field

$$
\mu_{u}^{\text {ind }}(\omega)=\alpha_{u v}(\omega) E_{v}(\omega)+\ldots,
$$

whose response-theory expression in the dipole approximation reads

$$
\boldsymbol{\alpha}(\omega)=\mathbf{X}_{a i}(\omega) \mathbf{P}_{i a}+\mathbf{Y}_{a i}(\omega) \mathbf{P}_{a i}
$$

It is related to the electric dipole-electric dipole response function via $\boldsymbol{\alpha}(\omega)=-\langle\langle\boldsymbol{\mu} ; \boldsymbol{\mu}\rangle\rangle_{\omega}$. The real part of the tensor describes dispersion while its imaginary part describes absorption. Specifically, in absorption experiments, the observed quantity is the dipole strength function

$$
S(\omega)=\frac{4 \pi \omega}{3 c} \Im[\operatorname{Tr} \boldsymbol{\alpha}(\omega)],
$$

where $\Im$ denotes the imaginary part and $c$ is the speed of light. The real part of $\boldsymbol{\alpha}(\omega)$ leads to the frequency-dependent index of refraction. In Eq. (36), $\mathbf{P}$ is the matrix representation of the electric dipole moment operator in $4 \mathrm{c}$ theory,

$$
\boldsymbol{P}_{p q}=-\left\langle\boldsymbol{\varphi}_{p}\left|\boldsymbol{\Sigma}_{0} \boldsymbol{r}\right| \boldsymbol{\varphi}_{q}\right\rangle
$$

\section{Electronic circular dichroism (ECD) and optical rotatory dispersion (ORD) spectroscopies}

The microscopic molecular property underpinning ORD and ECD spectra is the Rosenfeld tensor $^{70-72} \boldsymbol{\beta}$ that connects the induced electric dipole moment to the time derivative of a magnetic field $(\boldsymbol{B})$ as well as the induced magnetic moment to the time derivative of an electric field $(\boldsymbol{E})$

$$
\begin{aligned}
\mu_{u}^{\text {ind }}(\omega) & =\beta_{u v}(\omega) \dot{B}_{v}(\omega)+\ldots \\
m_{u}^{\text {ind }}(\omega) & =-\beta_{v u}(\omega) \dot{E}_{v}(\omega)+\ldots
\end{aligned}
$$

The ellipses stand for higher-order terms that can be neglected for isotropic samples in the linear response regime that are considered in this work. Eqs. (39) lead to two possible ways 
of calculating the $\boldsymbol{\beta}$ tensor quantum mechanically, either as an electric dipole response to a magnetic dipole perturbation (Eq. (39a)), or as a magnetic dipole response to an electric dipole perturbation (Eq. (39b)). In this work we adopt the second option and calculate the Rosenfeld tensor within linear damped response theory as

$$
\boldsymbol{\beta}(\omega)=-\frac{1}{\omega}\langle\langle\boldsymbol{m} ; \boldsymbol{\mu}\rangle\rangle_{\omega}=-\frac{1}{\omega}\left[\mathbf{X}_{a i}(\omega) \mathbf{M}_{i a}+\mathbf{Y}_{a i}(\omega) \mathbf{M}_{a i}\right] .
$$

In the realm of $4 \mathrm{c}$ relativistic theory, the matrix elements of the magnetic dipole moment matrix read

$$
\boldsymbol{M}_{p q}=-\frac{1}{4 c}\left\langle\boldsymbol{\varphi}_{p}\left|\boldsymbol{r}_{\mathrm{g}} \times \boldsymbol{\alpha}\right| \boldsymbol{\varphi}_{q}\right\rangle
$$

where $\boldsymbol{\alpha}$ is the vector of Dirac matrices in standard representation and $\boldsymbol{r}_{\mathrm{g}}=\boldsymbol{r}-\boldsymbol{R}_{\mathrm{g}}$ is the electron position operator relative to a fixed gauge origin, $\boldsymbol{R}_{\mathrm{g}}$.

ECD and ORD spectroscopies are based on differences in the complex index of refraction for left- and right-circularly polarized light in chiral molecules. The difference in the real (dispersive) part, i.e. circular birefringence, causes a rotation of the plane of polarization of the linearly polarized light passing through the medium. The difference in the imaginary (absorptive) part, i.e. circular dichroism, leads to the generation of ellipticity in the linearly polarized light. Optical rotation is measured either as the difference in refractive indices or as the angle of rotation of the linearly polarized light. Similarly, circular dichroism is measured either as the difference in extinction coefficients or as the induced ellipticity. The difference of the index of refraction $\Delta n(\omega)$ is related to the Rosenfeld tensor via

$$
\Delta n(\omega)=\frac{\omega \mathcal{N}}{3 c \epsilon_{0}} \operatorname{Tr}[\boldsymbol{\beta}(\omega)]
$$

where $\mathcal{N}$ is the particle concentration, $\epsilon_{0}$ is the vacuum permittivity, and SI units have been used. The differential extinction coefficient in ECD is related to the imaginary part of $\Delta n(\omega)$

$$
\Delta \varepsilon(\omega)=\frac{2 \omega N_{\mathrm{A}}}{c \mathcal{N} \ln (10)} \Im[\Delta n(\omega)]
$$

where $N_{\mathrm{A}}$ is the Avogadro constant, while the real part of $\Delta n(\omega)$ yields the angle of rotation in ORD

$$
\theta(\omega)=\frac{1}{2} \frac{l \rho \omega}{c} \Re[\Delta n(\omega)]
$$

where $l$ is the path length, $\rho$ is mass concentration and both Eqs. (43) and (44) use SI units. The differential extinction coefficient is commonly reported in $\mathrm{L} \mathrm{mol}^{-1} \mathrm{~cm}^{-1}$, leading to the 
conversion formula

$$
\Delta \varepsilon(\omega)=-10 \frac{2 N_{\mathrm{A}} e^{2} a_{0}}{\ln (10) c^{2} \epsilon_{0} m_{\mathrm{e}}} \omega^{\mathrm{AU}}\langle\langle\boldsymbol{m} ; \boldsymbol{\mu}\rangle\rangle_{\omega}^{\mathrm{AU}},
$$

where $a_{0}$ is the Bohr radius, $m_{\mathrm{e}}$ is the electron mass, and the superscript AU denotes quantities expressed in Hartree-based atomic units. ORD is customarily reported as specific rotation $[\alpha]_{\omega}$, i.e. the angle $\theta$ in degrees per unit mass concentration (in $\mathrm{g} \mathrm{cm}^{-1}$ ) per unit path length (in $\mathrm{dm}$ ), leading to the conversion formula

$$
[\alpha]_{\omega}=-10^{5} \frac{180}{\pi} \frac{N_{\mathrm{A}} e^{2} a_{0}}{2 M c^{2} \epsilon_{0} m_{\mathrm{e}}} \omega^{\mathrm{AU}}\langle\langle\boldsymbol{m} ; \boldsymbol{\mu}\rangle\rangle_{\omega}^{\mathrm{AU}} .
$$

where $M$ is the molecular mass in $\mathrm{g} \mathrm{mol}^{-1}$. The final units of $[\alpha]_{\omega}$ are $\operatorname{deg} \mathrm{dm}^{-1} \mathrm{~g}^{-1} \mathrm{~cm}^{3}$.

\section{RELATIVISTIC ITERATIVE SUBSPACE SOLVER: IMPLEMENTATION DETAILS}

The linear damped response equation in Eq. (16) can be viewed as a matrix equation where for realistic systems the matrix $\left[\mathbf{E}^{[2]}-(\omega+i \gamma) \mathbf{S}^{[2]}\right]$ is too large to be directly inverted, leaving iterative algorithms as the only viable option for its solution. ${ }^{61}$ The main idea of an iterative subspace algorithm is to express the solution $\mathbf{Z}$ as a linear combination in a subspace of so-called trial vectors, $\mathbf{T}_{m}$,

$$
\mathbf{Z} \approx \widetilde{\mathbf{Z}}=x_{m} \mathbf{T}_{m}
$$

and to solve for the complex expansion coefficients $x_{m}$. New trial vectors are dynamically added in the course of iterations until the Euclidean norm of a residue vector is smaller than a predefined threshold.

Specific details of a subspace solver depend on the level of theory used, specifically if spinorbit interaction is present or not, and the acceleration of its convergence can be achieved by a proper choice of parametrization. In $4 \mathrm{c}$ relativistic framework, the partitioning according to hermicity and time-reversal symmetry was advocated by Bast, Jensen and Saue ${ }^{20}$ in the context of the response eigenvalue equation, and later by Villaume, Saue and Norman ${ }^{44}$ in their work on the damped response equation. Our implementation utilizes the same idea, and the solution vector $\mathbf{Z}$ is divided into four symmetry components,

$$
\mathbf{Z}=\sum_{h t} \mathbf{Z}^{h t}=\mathbf{Z}^{++}+\mathbf{Z}^{+-}+\mathbf{Z}^{-+}+\mathbf{Z}^{--} .
$$


Here the superscripts denote symmetry $(+)$ or anti-symmetry $(-)$ with respect to hermitian $(h)$ or time-reversal symmetry $(t)$. Application of the individual terms in the damped response equation on $\mathbf{Z}^{\text {ht }}$ results in the following change of symmetries:

$$
\begin{aligned}
E_{a i, b j}^{[2]} Z_{b j}^{h t} & =Q_{a i}^{h t}, \\
\omega S_{a i, b j}^{[2]} Z_{b j}^{h t} & =Q_{a i}^{(-h) t}, \\
i \gamma S_{a i, b j}^{[2]} Z_{b j}^{h t} & =Q_{a i}^{h(-t)} .
\end{aligned}
$$

This symmetry mapping leads to the arrangement of the damped response equation into the matrix structure

$$
\left(\begin{array}{cccc}
\mathbf{E}^{[2]} & -i \gamma \mathbf{S}^{[2]} & -\omega \mathbf{S}^{[2]} & \mathbf{0} \\
-i \gamma \mathbf{S}^{[2]} & \mathbf{E}^{[2]} & \mathbf{0} & -\omega \mathbf{S}^{[2]} \\
-\omega \mathbf{S}^{[2]} & \mathbf{0} & \mathbf{E}^{[2]} & -i \gamma \mathbf{S}^{[2]} \\
\mathbf{0} & -\omega \mathbf{S}^{[2]} & -i \gamma \mathbf{S}^{[2]} & \mathbf{E}^{[2]}
\end{array}\right)\left(\begin{array}{l}
\mathbf{Z}^{++} \\
\mathbf{Z}^{+-} \\
\mathbf{Z}^{-+} \\
\mathbf{Z}^{--}
\end{array}\right)=\left(\begin{array}{l}
\mathbf{G}^{++} \\
\mathbf{G}^{+-} \\
\mathbf{G}^{-+} \\
\mathbf{G}^{--}
\end{array}\right)
$$

where the right-hand side is divided into the same symmetries as $\mathbf{Z}$.

Each $\mathbf{Z}^{\text {ht }}$ can be expressed in its own basis of symmetry-adapted trial vectors

$$
\mathbf{Z}^{h t} \equiv x_{m}^{h t} \mathbf{T}_{m}^{h t}
$$

The choice of hermicity and time-reversal symmetry leads to the following parametrization of the trial vectors

$$
\mathbf{T}^{h t} \equiv\left(\begin{array}{c}
\mathbf{W}^{t} \\
h \mathbf{W}^{t *}
\end{array}\right) .
$$

First, $\mathbf{T}^{+t}$ and $\mathbf{T}^{-t}$ are referred to as hermitian and anti-hermitian trial vectors, respectively, and the motivation for their structure and introduction stems from the solution of the response equation for frequencies $\omega$ and $-\omega \cdot{ }^{43}$ Second, after rearrangement of the vector $\mathbf{W}^{t} \in \mathbb{C}^{N_{\mathrm{v}} \cdot N_{\mathrm{o}}}$ to the matrix form $\mathbf{W}^{t} \in \mathbb{C}^{N_{\mathrm{v}} \times N_{\mathrm{o}}}$, it gains the specific internal structure

$$
\mathbf{W}^{t} \equiv\left(\begin{array}{cc}
\mathbf{U} & \mathbf{V} \\
-t \mathbf{V}^{*} & t \mathbf{U}^{*}
\end{array}\right) .
$$

The motivation for the use of time-reversal symmetric $\mathbf{T}^{h+}$ and anti-symmetric $\mathbf{T}^{h-}$ trial vectors can be rationalized as follows: for the closed-shell molecular systems considered in this work, the canonical MOs are obtained as degenerate Kramers partners, $\left\{\boldsymbol{\varphi}_{p}, \overline{\boldsymbol{\varphi}}_{p}\right\}$, 
with $\bar{\varphi}_{p}$ obtained by time conjugation of $\boldsymbol{\varphi}_{p}$. As a consequence, we can construct $\mathrm{U}, \mathrm{V} \in$ $\mathbb{C}^{N_{\mathrm{V}} / 2 \times N_{\mathrm{o}} / 2}$ where $\mathbf{U}$ is represented in an unbarred-unbarred basis while $\mathbf{V}$ in an unbarredbarred basis. Thanks to this specific arrangement, in the case of zero damping factor, only time-reversal symmetric trial vectors $\mathbf{T}^{h+}$ contribute to the expansion in Eq. (51). Since every complex matrix $\mathbb{C}^{N_{\mathrm{v}} \times N_{\mathrm{o}}}$ can be decomposed into its time-reversal symmetric and antisymmetric part, for the case of non-zero damping factor, it is sufficient to add time-reversal anti-symmetric $\mathbf{T}^{h-}$ trial vectors.

The parametrization of trial vectors should be robust with respect to a phase change in the MOs, $\boldsymbol{\varphi} \mapsto e^{i \alpha} \boldsymbol{\varphi}$, where $\alpha$ is a time-independent parameter. The phase change in the orbital translates into the $\mathbf{d}$-coefficients via Eq. (6) as $\mathbf{d} \mapsto e^{i \alpha} \mathbf{d}$ and to the solution vector via Eq. (14) as

$$
\mathbf{Z}=\left(\begin{array}{c}
\mathbf{X} \\
\mathbf{Y}
\end{array}\right) \mapsto\left(\begin{array}{c}
\mathbf{X} e^{i \alpha} \\
\mathbf{Y} e^{-i \alpha}
\end{array}\right)
$$

Therefore, while the real part of the phase factor does not affect the ht symmetries, its imaginary part does. In the most extreme case, the change amounts to a multiplication by the imaginary unit that flips both the hermicity and the time-reversal symmetry. This poses a problem in a finite basis set since in two consequent iterations the basis for $\mathbf{Z}$ is different, allowing for a phase change. If two components of the solution that have both symmetries opposite are expressed in a different basis, the solution of the previous iteration will not be captured in the next. This can be resolved by introducing new trial vectors $\overline{\mathbf{T}}_{m}^{++}$and $\overline{\mathbf{T}}_{m}^{-+}$ defined as

$$
\overline{\mathbf{T}}_{m}^{++} \equiv i \mathbf{T}_{m}^{--}, \quad \overline{\mathbf{T}}_{m}^{-+} \equiv i \mathbf{T}_{m}^{+-} .
$$

and uniting them with $\mathbf{T}_{m}^{++}$and $\mathbf{T}_{m}^{-+}$into two sets of basis vectors characterized by their hermicity only:

$$
\begin{aligned}
& \left\{\mathbf{b}_{m}^{+}\right\}=\left\{\mathbf{T}_{m}^{++}\right\} \oplus\left\{\overline{\mathbf{T}}_{m}^{++}\right\}, \\
& \left\{\mathbf{b}_{m}^{-}\right\}=\left\{\mathbf{T}_{m}^{-+}\right\} \oplus\left\{\overline{\mathbf{T}}_{m}^{-+}\right\}
\end{aligned}
$$

In these new bases, the final expansion of $\mathbf{Z}^{h t}$ is performed as

$$
\begin{array}{ll}
\mathbf{Z}^{++}=x_{m}^{++} \mathbf{b}_{m}^{+}, & \mathbf{Z}^{--}=x_{m}^{--} i \mathbf{b}_{m}^{+} \\
\mathbf{Z}^{-+}=x_{m}^{-+} \mathbf{b}_{m}^{-}, & \mathbf{Z}^{+-}=x_{m}^{+-} i \mathbf{b}_{m}^{-}
\end{array}
$$


Note that other choices for $\overline{\mathbf{T}}$ are possible, for example defining $\overline{\mathbf{T}}_{m}^{+-} \equiv i \mathbf{T}_{m}^{-+}$, which leads to two sets of basis vectors characterized by time-reversal symmetry instead of hermicity as adopted here. In principle this alternative should lead to similar solver robustness but we have not pursued this further. Additionally, the bases defined by vectors $\mathbf{b}^{h}$ are in a given iteration larger that the bases defined by $\mathbf{T}^{h t}$ meaning that expansion in Eqs. (57) also offers better flexibility for convergence than expansion in Eq. (51). However, if the barred vectors are linearly dependent on already existing vectors, these are not added to the final basis and hence computational and memory requirements are reduced.

\section{Algorithm}

The iterative solver for the damped response equation in the form of Eq. (50) was implemented in the ReSpect $\operatorname{program}^{58}$ and proceeds in the following steps:

1. The right-hand side of Eq. (50) is constructed, which for the electric dipole perturbation considered in this work has non-zero component for $\mathrm{G}^{++}$only.

2. The initial guess for each solution component $\mathbf{Z}^{\text {ht }}$ is obtained as

$$
\mathbf{Z}^{h t(0)}=\left[\widetilde{\mathbf{M}}^{-1} \mathbf{G}\right]^{h t}
$$

where $\widetilde{\mathbf{M}}^{-1}$ is the inverse of a matrix constructed by approximating the Hessian $E_{a i, b j}^{[2]} \approx$ $\delta_{i j} \delta_{a b} \epsilon_{a i}$ in Eq. (50). For compactness, we have introduced the notation $\epsilon_{a i} \equiv \epsilon_{a}-\epsilon_{i}$. Each block in matrix $\widetilde{\mathbf{M}}$ is now diagonal and its inversion can be performed directly with the result

$$
\widetilde{\mathbf{M}}^{-1} \equiv \mathcal{F}^{-1}\left(\begin{array}{cccc}
\mathcal{A} & \mathcal{B} & \mathcal{C} & \mathcal{D} \\
\mathcal{B} & \mathcal{A} & \mathcal{D} & \mathcal{C} \\
\mathcal{C} & \mathcal{D} & \mathcal{A} & \mathcal{B} \\
\mathcal{D} & \mathcal{C} & \mathcal{B} & \mathcal{A}
\end{array}\right)
$$


where

$$
\begin{aligned}
& \mathcal{A}_{a i, a i}=\epsilon_{a i}\left(\epsilon_{a i}^{2}+\gamma^{2}-\omega^{2}\right) \\
& \mathcal{B}_{a i, a i}=i \gamma\left(\epsilon_{a i}^{2}+\gamma^{2}+\omega^{2}\right) \\
& \mathcal{C}_{a i, a i}=-\omega\left(-\epsilon_{a i}^{2}+\gamma^{2}+\omega^{2}\right) \\
& \mathcal{D}_{a i, a i} \equiv 2 i \gamma \omega \epsilon_{a i} \\
& \mathcal{F}_{a i, a i}=\epsilon_{a i}^{4}+2 \epsilon_{a i}^{2}\left(\gamma^{2}-\omega^{2}\right)+\left(\gamma^{2}+\omega^{2}\right)^{2} .
\end{aligned}
$$

3. For an iteration $n$, trial vectors $\mathbf{T}^{h t(n)}$ are generated either by taking $\mathbf{Z}^{h t(0)}$ if $n=0$, or by preconditioning the residue vector $\mathbf{R}^{(n-1)}$ if $n>0$

$$
\mathbf{T}^{h t(n)}=\left(\widetilde{\mathbf{M}}^{-1} \mathbf{R}^{(n-1)}\right)^{h t}
$$

where the symmetry components of the residue vector are

$$
\mathbf{R}^{h t(n)}=\mathbf{G}^{h t}-\mathbf{E}^{[2]} \mathbf{Z}^{h t(n)}+i \gamma \mathbf{S}^{[2]} \mathbf{Z}^{h-t(n)}+\omega \mathbf{S}^{[2]} \mathbf{Z}^{-h t(n)} .
$$

4. Trial vectors from the previous step are orthogonalized by means of the modified GramSchmidt procedure and used to construct bases $\mathbf{b}^{+}$and $\mathbf{b}^{-}$according to Eqs. (56). The orthogonalization is performed with respect to vectors already present in the $\mathbf{b}^{h}$ bases. Only those trial vectors with norm above a pre-defined threshold are normalized and added to bases $\mathbf{b}^{h}$.

5. Eq. (50) is projected onto the subspace defined by vectors $\mathbf{b}^{h}$, followed by exchanging the second and third columns and rows, which finally yields the symmetric reduced equation

$$
\left(\begin{array}{cccc}
\mathbf{E}_{++}^{[2]} & -\omega \mathbf{S}_{+-}^{[2]} & \gamma \mathbf{S}_{+-}^{[2]} & \mathbf{0} \\
-\omega \mathbf{S}_{-+}^{[2]} & \mathbf{E}_{--}^{[2]} & \mathbf{0} & \gamma \mathbf{S}_{-+}^{[2]} \\
\gamma \mathbf{S}_{-+}^{[2]} & \mathbf{0} & -\mathbf{E}_{--}^{[2]} & \omega \mathbf{S}_{-+}^{[2]} \\
\mathbf{0} & \gamma \mathbf{S}_{+-}^{[2]} & \omega \mathbf{S}_{+-}^{[2]} & -\mathbf{E}_{++}^{[2]}
\end{array}\right)\left(\begin{array}{c}
\boldsymbol{x}^{++} \\
\boldsymbol{x}^{-+} \\
\boldsymbol{x}^{+-} \\
\boldsymbol{x}^{--}
\end{array}\right)=\left(\begin{array}{c}
\mathbf{G}_{+} \\
\mathbf{G}_{-} \\
-i \mathbf{G}_{-} \\
-i \mathbf{G}_{+}
\end{array}\right),
$$

where $\mathbf{E}_{h h^{\prime}}^{[2]} \equiv\left\langle\mathbf{b}^{h}\left|\mathbf{E}^{[2]}\right| \mathbf{b}^{h^{\prime}}\right\rangle$ and $\mathbf{S}_{h h^{\prime}}^{[2]} \equiv\left\langle\mathbf{b}^{h}\left|\mathbf{S}^{[2]}\right| \mathbf{b}^{h^{\prime}}\right\rangle$ are much smaller matrices than $\mathbf{E}^{[2]}$ and $\mathbf{S}^{[2]}$ in Eq. (50) of dimensions $N_{h} \times N_{h^{\prime}}$, with $N_{h}$ referring to the number of basis vectors of symmetry $h$. Similarly, the right-hand side of Eq. (50) is defined as $\mathbf{G}_{h} \equiv\left\langle\mathbf{b}^{h} \mid \mathbf{G}^{h}\right\rangle$. From a symmetry analysis, one finds that all elements of Eq. (63) are purely real. The reduced equation is solved as a low-dimensional linear system by the LAPACK's LU factorization routine ${ }^{73}$. 
6. For iteration $n$, the solution vectors $\mathbf{Z}^{h t(n)}$ are constructed according to Eq. (57) and used to calculate residues $\mathbf{R}^{h t(n)}$ according to Eq. (62). Iterations terminate if the Euclidean norm $\left\|\mathbf{R}^{(n)}\right\|$ of the total residue $\mathbf{R}^{(n)}=\sum_{h t}\left|\mathbf{R}^{h t(n)}\right|$ is below a predefined threshold, otherwise the algorithm continues by repeating steps 3-6.

The methodology allows for several frequencies to be treated simultaneously, which gives a great advantage as it significantly accelerates convergence. Each frequency can contribute up to 4 basis vectors per iteration to the trial subspace and its corresponding solution vector is expanded in a common basis composed of trial vectors generated by all the frequencies.

\section{COMPUTATIONAL DETAILS}

The systems selected in this study are $(2 \mathrm{R}, 3 \mathrm{R})$-dimethylchalcogeniranes $\mathrm{C}_{4} \mathrm{H}_{8} \mathrm{X}(\mathrm{X}=\mathrm{O}$, $\mathrm{S}, \mathrm{Se}, \mathrm{Te}, \mathrm{Po}, \mathrm{Lv})$ and the chiral tris-phentantroline complex cations $\Delta-\left[\mathrm{M}(\mathrm{phen})_{3}\right]^{2+}(\mathrm{M}=$ $\mathrm{Fe}, \mathrm{Ru}, \mathrm{Os})$. For dimethylchalcogeniranes, geometries were taken from the supplementary material of Ref. 54, and for $\left[\mathrm{M}(\mathrm{phen})_{3}\right]^{2+}$ from the supplementary information of Ref. 74 .

The calculations of polarizabilities, EAS, ECD and ORD spectra were performed with the ReSpect program ${ }^{58}$ employing a newly implemented library for 1c and 4c linear damped response theory. The development and implementation of the 4c methodology is described in Section II and the 1c implementation closely follows work of Kauczor and Norman ${ }^{75}$. The property calculations utilized the $\mathrm{PBE}^{76-78}$ and $\mathrm{PBE}^{76-79}$ exchange-correlation functionals. The numerical integration of the noncollinear exchange-correlation potential and kernel was done with an adaptive molecular grid of medium size (program default). In 4c calculations, atomic nuclei of finite size were approximated by a Gaussian charge distribution model ${ }^{80}$ and four-center two-electron repulsion integrals were treated within an atom-pair approximation where all integrals over the atom-centered small-component basis functions $X^{\mathrm{S}}$ are discarded unless the bra and ket basis pairs share the same origin, i.e., $\left[X_{A}^{\mathrm{S}} X_{B}^{\mathrm{S}} \mid X_{C}^{\mathrm{S}} X_{D}^{\mathrm{S}}\right] \delta_{A B} \delta_{C D}$, where $\delta$ is the Kronecker delta function over atomic centers $A, B, C$, and $D$. Calculations involving pure DFT functional PBE have been accelerated by the resolution-of-the-identity technique for the Coulomb term (RI-J) described in Ref. 54.

All systems were treated using uncontracted all-electron GTO basis sets. The specific bases used were Dyall's augmented cVDZ basis ${ }^{81-83}$ for Te, Po, and Lv and Dunning's augmented cc-pVDZ basis ${ }^{84-86}$ for other elements in the dimethylchalcogeniranes, and Dyall's 
cVDZ basis ${ }^{87-90}$ for Fe, Ru, and Os and Dunning's cc-pVDZ ${ }^{91}$ for other elements in the trisphenantroline complexes. The auxiliary basis sets for the RI-J procedure were generated by an adjusted even-tempered algorithm ${ }^{92}$ (and are available in the supplementary information).

The dimethylchalcogenirane spectra calculated with the PBE functional were compared with RT-TDDTF results also calculated with ReSpect and reported in Ref. 54. This was reflected in the damped linear response settings by using a frequency step of $0.038 \mathrm{eV}$ in the spectral range from 0 to $12 \mathrm{eV}$ with the damping factor $\gamma=0.004 \mathrm{au}$. Novel spectra of dimethylchalcogeniranes with the PBE0 functional and all spectra of the metal complexes were calculated on frequency points distributed every $0.05 \mathrm{eV}$ in the spectral ranges from 0 to $10 \mathrm{eV}$ and from $1.5 \mathrm{eV}$ to $5.5 \mathrm{eV}$, respectively. All linear response calculations employed the multi-frequency solver with $20-50$ points treated simultaneously. Excitations to virtual negative-energy states were neglected in damped response calculations at the $4 \mathrm{c}$ relativistic level of theory.

All calculations presented in this paper assume the gauge origin to be placed in the centre of mass of the molecule. We note that the present methodology is gauge dependent, this dependence for ECD and ORD having been studied at the non-relativistic and relativistic levels of theory ${ }^{93-95}$, and we have not explored this further in this work.

\section{RESULTS AND DISCUSSION}

To test the validity of the $1 \mathrm{c}$ and $4 \mathrm{c}$ linear damped response (DR) implementations presented here, we first evaluate electronic absorption (EAS) and circular dichroism (ECD) spectra for the dimethylchalcogenirane series $\mathrm{C}_{4} \mathrm{H}_{8} \mathrm{X}(\mathrm{X}=\mathrm{O}, \mathrm{S}, \mathrm{Se}, \mathrm{Te}, \mathrm{Po}, \mathrm{Lv})$ and compare the results with reference data obtained with the real-time TDDFT (RT-TDDFT) methodology presented earlier ${ }^{54}$. The final spectra resulting from these two methods should be identical, since we impose a weak-field regime of RT-TDDFT in addition to choosing computational settings for the damped response solver that match those used in the previous RT-TDDFT work. The benchmark series starts with dimethyloxirane, a prototypical chiral molecule often used in benchmark chiroptical studies, extended further to the heavier analogues with S, Se, Te, Po, and Lv as heteroatoms (the latter three being model systems) to gauge the relativistic effects on the EAS and ECD spectra arising from the presence of the heavy elements. 
The resulting EAS and ECD spectra are depicted in Fig. 1 for the Se-Lv systems, whereas the corresponding spectra for the light $\mathrm{O}$ and $\mathrm{S}$ analogues, as well as additional molecular properties (linear electric-dipole polarizabilities and optical rotations) for all molecules, are available in the supplementary material. While there is practically no difference between the $1 \mathrm{c}$ and $4 \mathrm{c}$ results for the two lightest systems, the differences in both spectra become noticeable starting with Se. For the Po- and Lv-substituted systems, the 1c results cannot be considered even as an approximation of the relativistic results. Particularly, for the Po system, the 1c ECD spectra resemble the mirror image of the relativistic spectra in a region from approximately 4.5 to $7.0 \mathrm{eV}$, leading to an incorrect stereodescriptor assignment if relativistic effects are neglected. This corroborates the conclusion drawn in the previous RT-TDDFT study ${ }^{54}$ that the neglect of relativity may lead to incorrect determination of absolute configuration in compounds containing heavy elements. Nevertheless, the good performance of the 1c non-relativistic methods for the Te system is somewhat surprising since in general it is assumed that for 5th-row elements the inclusion of relativistic effects is mandatory.

Furthermore, we extended the treatment of the studied systems to a hybrid functional, specifically PBE0. The figures resulting spectra are available in the supplementary material. The difference between PBE0 and PBE amounts to a shift in the spectra for lower frequencies, while more notable differences arise as the frequency increases. The apparent change of sign due to relativity for dimethylpolonirane persists also at the PBE0 level of theory.

Finally, visual inspection of the EAS and ECD spectra in Fig. 1 hints to an excellent agreement between the damped response and real-time TDDFT approaches. As a quantitative measure we report the relative mean absolute difference of corresponding spectral functions. The results are presented in Table I. An alternative reading of the numbers in Table I is as a measure of importance of higher-order contributions present in RT-TDDFT method for a given field strength.

In order to demonstrate that the ReSpect implementation of 4c damped response theory involving pure and hybrid exchange-correlation functionals is applicable to chemically relevant systems, we calculated the EAS and ECD spectra of series of larger chiral transition metal complexes, $\left[\mathrm{M}(\mathrm{phen})_{3}\right]^{2+}(\mathrm{M}=\mathrm{Fe}, \mathrm{Ru}, \mathrm{Os})$. These systems have been subjects of earlier studies using TDDFT and approximative scalar ZORA Hamiltonian ${ }^{74,96,97}$. These studies showed that ECD spectra of these systems were only weakly affected by the choice of 
TABLE I: Relative mean absolute difference (RMAD) between 4c linear damped response (DR) and 4c real-time (RT) TDDFT methodologies for ECD (differential extinction coeffcient) and EAS (dipole strength function) spectral functions in dimethylchalcogeniranes $\mathrm{C}_{4} \mathrm{H}_{8} \mathrm{X}(\mathrm{X}=\mathrm{O}, \mathrm{S}, \mathrm{Se}, \mathrm{Te}, \mathrm{Po}, \mathrm{Lv})$.

\begin{tabular}{ccc}
\hline \hline Heteroatom & $\mathrm{ECD}^{\mathrm{a}}$ & $\left.\mathrm{EAS}^{\mathrm{a}}\right)$ \\
\hline $\mathrm{O}$ & $1.23 \%$ & $0.14 \%$ \\
$\mathrm{~S}$ & $0.35 \%$ & $0.06 \%$ \\
$\mathrm{Se}$ & $0.53 \%$ & $0.43 \%$ \\
$\mathrm{Te}$ & $0.97 \%$ & $0.22 \%$ \\
$\mathrm{Po}$ & $4.30 \%$ & $1.03 \%$ \\
$\mathrm{Lv}$ & $4.50 \%$ & $1.10 \%$ \\
\hline \hline
\end{tabular}

a) $\mathrm{RMAD}=\frac{\sum_{i}\left|\operatorname{DR}\left(\omega_{i}\right)-\mathrm{RT}\left(\omega_{i}\right)\right|}{\sum_{j}\left|\operatorname{RT}\left(\omega_{j}\right)\right|} \cdot 100 \%$

basis set and DFT functional (within the same pure or hybrid class of functionals). Furthermore, solvent effects are negligible, whereas relativistic effects are noticeable. In contrast to the previous studies, however, the present work uses $\Delta$ isomers with geometries taken from the supporting information of Ref. 74 .

The results of both the EAS and ECD spectra calculations for these systems are presented in Figure 2, where ECD spectra have been multiplied by a factor of 6 in the region between 1.5 and $4.0 \mathrm{eV}$ to amplify the lower-intensity part of the spectra (the same scaling was applied in Ref. 74). In general, there is a good agreement between both the EAS and ECD spectra obtained at the $1 \mathrm{c}$ and $4 \mathrm{c}$ levels of theory with differences becoming more pronounced for the complexes with the heavier central atoms. Specifically, the low-frequency peaks (below $4.0 \mathrm{eV}$ ) are affected more and the relativistic effects appear as shifts and new peaks between 2 and $3 \mathrm{eV}$. In contrast, the strong peak centered at $4.5 \mathrm{eV}(\mathrm{PBE})$ and $5.0 \mathrm{eV}$ (PBE0), respectively, remains unchanged. To investigate this dependence, we applied the analysis described in Section IIC to determine the nature of the spectral lines in terms of MO transitions with examples presented in Fig 3. This analysis showed that the signals below $4.0 \mathrm{eV}$ originate from metal-to-ligand charge transfer (MLCT) excitations from d-type spinors of the central atom to ligand orbitals, whereas the intense higher-energy transition results from a ligand to ligand excitation, thus confirming the conclusions of Ref. 74. The 
$\mathrm{MO}$ origin of transitions also explains the dependence of the low-frequency MLCT peaks on relativity, since the orbitals of the heavy central atom are expected to be more affected by relativity than the light ligand atoms. The analysis was performed both on EAS and ECD spectra and yields identical results for transitions that are active in both spectroscopies.

A more noticeable change in the spectra was achieved by a switch to the hybrid functional, as also reported in the previous study by Rudolph and Autschbach ${ }^{97}$. They also compared the calculated spectra to experiment and noted a good agreement for the hybrid functionals and mixed performance of the pure functionals. Based on our results it is clear that for the studied complexes the final spectra are much less influenced by the relativistic effects (both scalar and spin-orbit) than by the choice of pure or hybrid DFT functional, respectively.

\section{SUMMARY AND OUTLOOK}

We have presented a detailed derivation and implementation of relativistic four-component linear damped response TDDFT (or complex polarization propagator) into the ReSpect program, and its application to the calculation of frequency-dependent linear dipole polarizability, electronic absorption, natural circular dichroism, and optical rotation. The method prevents divergencies at near-resonant frequencies through a damping parameter and allows simultaneous description of both absorption and dispersion processes, particularly in highfrequency spectral regions that may prove expensive to access with real-time TDDFT and in high density-of-states cases that are challenging for eigenvalue response equation. To the best of our knowledge, this work reports the first 4c damped linear response TDDFT results of ECD and ORD presented in the literature.

The damped response equation is solved using a multi-frequency iterative subspace solver utilizing the decomposition of the equation into hermitian and anti-hermitian, and timereversal symmetric and anti-symmetric parts. This partitioning leads to robust convergence in all systems studied for all frequencies. Electron correlation is treated at the non-collinear Kohn-Sham DFT level of theory. For the case of pure DFT functionals, the current implementation enables acceleration through the resolution-of-the-identity technique for the Coulomb term.

Combined with our earlier work, ${ }^{17,52}$ the ReSpect program now possesses three distinct TDDFT approaches (real-time TDDFT, response eigenvalue equation and damped 
response theory) to dynamical linear response property calculations at the non-relativistic one-component and the relativistic four-component level of theory. This makes ReSpect the only relativistic quantum chemistry computer program featuring all three approaches, and allows the users to select the most suitable method to address a particular chemical problem.

\section{SUPPLEMENTARY MATERIAL}

See supplementary material auxiliary basis sets, and additional linear electric dipole polarizabilities, EAS, ECD, and ORD spectra.

\section{ACKNOWLEDGMENTS}

The work has received support from the Research Council of Norway through a Center of Excellence Grant (Grant No. 262695). LK acknowledges support from the Grant Agency of the Ministry of Education of the Slovak Republic and Slovak Academy of Sciences VEGA (Grant No. 1/0507/17). Computer time was provided by the Norwegian Supercomputer Program NOTUR (Grant No. NN4654K) as well as by the Large Infrastructures for Research, Experimental Development and Innovations project "IT4Innovations National Supercomputing Center - LM2015070" (Project No. OPEN-15-32) supported by The Ministry of Education, Youth and Sports of the Czech Republic. SK acknowledges financial support from the Slovak Grant Agencies VEGA and APVV (contract no. 2/0116/17 and APVV-15-0726). LK would like to thank Marius Kadek and Patrick Norman for helpful discussions.

\section{REFERENCES}

${ }^{1}$ C. A. Ullrich, Time-Dependent Density-Functional Theory: Concepts and Applications (OUP Oxford, 2011).

${ }^{2}$ M. A. Marques and E. K. Gross, Annu. Rev. Phys. Chem. 55, 427 (2004).

${ }^{3}$ F. Neese, Coord. Chem. Rev. 253, 526 (2009).

${ }^{4}$ M. E. Casida and M. Huix-Rotllant, Annu. Rev. Phys. Chem. 63, 287 (2012).

${ }^{5}$ P. Pyykkö, Annu. Rev. Phys. Chem. 63, 45 (2012).

${ }^{6}$ W. Liu, Mol. Phys. 108, 1679 (2010). 
${ }^{7}$ T. Saue, ChemPhysChem 12, 3077 (2011).

${ }^{8}$ M. E. Casida, in Recent Advances In Density Functional Methods: (Part I) (World Scientific, 1995) pp. 155-192.

${ }^{9}$ A. Dreuw and M. Head-Gordon, Chem. Rev. 105, 4009 (2005).

${ }^{10}$ M. E. Casida, J. Mol. Struct.: THEOCHEM 914, 3 (2009).

${ }^{11}$ T. Helgaker, S. Coriani, P. Jørgensen, K. Kristensen, J. Olsen, and K. Ruud, Chem. Rev. 112, 543 (2012).

${ }^{12}$ J. Brabec, L. Lin, M. Shao, N. Govind, C. Yang, Y. Saad, and E. G. Ng, J. Chem. Theory Comput. 11, 5197 (2015).

${ }^{13}$ F. Furche, B. T. Krull, B. D. Nguyen, and J. Kwon, J. Chem. Phys. 144, 174105 (2016).

${ }^{14}$ C. Huang, W. Liu, Y. Xiao, and M. R. Hoffmann, J. Comput. Chem. 38, 2481 (2017).

${ }^{15}$ J. M. Kasper, D. B. Williams-Young, E. Vecharynski, C. Yang, and X. Li, J. Chem. Theory Comput. 14, 2034 (2018).

${ }^{16}$ C. Huang and W. Liu, J. Comput. Chem. 40, 1023 (2019).

${ }^{17}$ S. Komorovsky, P. Cherry, and M. Repisky, submitted (2019).

${ }^{18}$ P. Norman and A. Dreuw, Chem. Rev. 118, 7208 (2018).

${ }^{19}$ T. F. Stetina, J. M. Kasper, and X. Li, J. Chem. Phys. 150, 234103 (2019).

${ }^{20}$ R. Bast, H. J. A. Jensen, and T. Saue, Int. J. Quantum Chem. 109, 2091 (2009).

${ }^{21}$ G. Scalmani and M. J. Frisch, J. Chem. Theory Comput. 8, 2193 (2012).

${ }^{22}$ F. Egidi, S. Sun, J. J. Goings, G. Scalmani, M. J. Frisch, and X. Li, J. Chem. Theory Comput. 13, 2591 (2017).

${ }^{23}$ J. Gao, W. Liu, B. Song, and C. Liu, J. Chem. Phys. 121, 6658 (2004).

${ }^{24}$ J. Gao, W. Zou, W. Liu, Y. Xiao, D. Peng, B. Song, and C. Liu, J. Chem. Phys. 123, $054102(2005)$.

${ }^{25}$ Z. Li, B. Suo, Y. Zhang, Y. Xiao, and W. Liu, Mol. Phys. 111, 3741 (2013).

${ }^{26}$ M. Kühn and F. Weigend, J. Chem. Theory Comput. 9, 5341 (2013).

${ }^{27}$ F. Egidi, J. J. Goings, M. J. Frisch, and X. Li, J. Chem. Theory Comput. 12, 3711 (2016).

${ }^{28}$ D. Peng, W. Zou, and W. Liu, J. Chem. Phys. 123, 144101 (2005).

${ }^{29}$ F. Wang and T. Ziegler, J. Chem. Phys. 123, 154102 (2005).

${ }^{30}$ F. Wang, T. Ziegler, E. van Lenthe, S. van Gisbergen, and E. J. Baerends, J. Chem. Phys. 122, 204103 (2005).

${ }^{31}$ W. Liu and Y. Xiao, Chem. Soc. Rev. 47, 4481 (2018). 
${ }^{32}$ Y. Imamura, M. Kamiya, and T. Nakajima, Chem. Phys. Lett. 635, 152 (2015).

${ }^{33}$ L. Grajciar, J. Phys. Chem. C 120, 27050 (2016).

${ }^{34}$ Y. Gao, B. Wang, Y. Lei, B. K. Teo, and Z. Wang, Nano Res. 9, 622 (2016).

${ }^{35}$ K. Mori, T. Goumans, E. Van Lenthe, and F. Wang, Phys. Chem. Chem. Phys. 16, 14523 (2014).

${ }^{36}$ M. Kühn and F. Weigend, J. Chem. Phys. 141, 224302 (2014).

${ }^{37}$ T. Fransson, T. Saue, and P. Norman, J. Chem. Theory Comput. 12, 2324 (2016).

${ }^{38}$ J. Oddershede, P. Jørgensen, and D. L. Yeager, Comput. Phys. Rep. 2, 33 (1984).

${ }^{39}$ P. Norman, D. M. Bishop, H. J. A. Jensen, and J. Oddershede, J. Chem. Phys. 115, 10323 (2001).

${ }^{40}$ P. Norman, D. M. Bishop, H. J. A. Jensen, and J. Oddershede, J. Chem. Phys. 123, 194103 (2005).

${ }^{41}$ P. Norman, A. Jiemchooroj, and B. E. Sernelius, J. Chem. Phys. 118, 9167 (2003).

${ }^{42}$ A. Devarajan, A. Gaenko, and J. Autschbach, J. Chem. Phys. 130, 194102 (2009).

${ }^{43}$ T. Saue and H. A. Jensen, J. Chem. Phys. 118, 522 (2003).

${ }^{44}$ S. Villaume, T. Saue, and P. Norman, J. Chem. Phys. 133, 064105 (2010).

${ }^{45}$ N. Gaston, P. Schwerdtfeger, T. Saue, and J. Greif, J. Chem. Phys. 124, 044304 (2006).

${ }^{46}$ T. Fransson, D. Burdakova, and P. Norman, Phys. Chem. Chem. Phys. 18, 13591 (2016).

${ }^{47}$ C. South, A. Shee, D. Mukherjee, A. K. Wilson, and T. Saue, Phys. Chem. Chem. Phys. 18, 21010 (2016).

${ }^{48}$ D. Sulzer, P. Norman, and T. Saue, Mol. Phys. 110, 2535 (2012).

${ }^{49}$ J. Theilhaber, Phys. Rev. B 46, 12990 (1992).

${ }^{50}$ K. Yabana and G. Bertsch, Phys. Rev. B 54, 4484 (1996).

${ }^{51}$ J. J. Goings, P. J. Lestrange, and X. Li, Wiley Interdiscip. Rev. Comput. Mol. Sci. 8, 1 (2018).

${ }^{52}$ M. Repisky, L. Konecny, M. Kadek, S. Komorovsky, O. L. Malkin, V. G. Malkin, and K. Ruud, J. Chem. Theory Comput. 11, 980 (2015).

${ }^{53}$ M. Kadek, L. Konecny, B. Gao, M. Repisky, and K. Ruud, Phys. Chem. Chem. Phys. 17, 22566 (2015).

${ }^{54}$ L. Konecny, M. Kadek, S. Komorovsky, K. Ruud, and M. Repisky, J. Chem. Phys. 149, 204104 (2018). 
${ }^{55}$ J. J. Goings, J. M. Kasper, F. Egidi, S. Sun, and X. Li, J. Chem. Phys. 145, 104107 (2016).

${ }^{56}$ J. M. Kasper, P. J. Lestrange, T. F. Stetina, and X. Li, J. Chem. Theory Comput. 14, 1998 (2018).

${ }^{57}$ L. Konecny, M. Kadek, S. Komorovsky, O. L. Malkina, K. Ruud, and M. Repisky, J. Chem. Theory Comput. 12, 5823 (2016).

${ }^{58}$ ReSpect 5.2.0 (2019), relativistic spectroscopy DFT program of authors M. Repisky, S. Komorovsky, V. G. Malkin, O. L. Malkina, M. Kaupp, K. Ruud, with contributions from R. Bast, R. Di Remigio, U. Ekstrom, M. Kadek, S. Knecht, L. Konecny, E. Malkin, I. Malkin Ondik (see http://www.respectprogram.org).

${ }^{59}$ P. Norman, K. Ruud, and T. Saue, Principles and practices of molecular properties: Theory, modeling, and simulations (Wiley Online Library, 2018).

${ }^{60}$ J. Olsen and P. Jørgensen, J. Chem. Phys. 82, 3235 (1985).

${ }^{61}$ J. Kauczor, P. Jørgensen, and P. Norman, J. Chem. Theory Comput. 7, 1610 (2011).

${ }^{62}$ G. P. Chen, V. K. Voora, M. M. Agee, S. G. Balasubramani, and F. Furche, Annu. Rev. Phys. Chem. 68, 421 (2017).

${ }^{63}$ S. Hirata and M. Head-Gordon, Chem. Phys. Lett. 314, 291 (1999).

${ }^{64}$ S. Komorovsky, M. Repisky, O. L. Malkina, V. G. Malkin, I. Malkin Ondik, and M. Kaupp,

J. Chem. Phys. 128, 104101 (2008).

${ }^{65}$ S. Komorovsky, M. Repisky, E. Malkin, T. B. Demissie, and K. Ruud, J. Chem. Theory Comput. 11, 3729 (2015).

${ }^{66}$ M. Kadek, M. Repisky, and K. Ruud, Phys. Rev. B 99, 205103 (2019).

${ }^{67}$ C. van Wüllen, J. Comput. Chem. 23, 779 (2002).

${ }^{68}$ F. Wang and T. Ziegler, J. Chem. Phys. 121, 12191 (2004).

${ }^{69}$ F. Wang and T. Ziegler, J. Chem. Phys. 122, 074109 (2005).

${ }^{70}$ L. Rosenfeld, Z. Phys. 52, 161 (1929).

${ }^{71}$ E. U. Condon, Rev. Mod. Phys. 9, 432 (1937).

${ }^{72}$ L. D. Barron, Molecular Light Scattering and Optical Activity (Cambridge University Press, 2004).

${ }^{73}$ E. Anderson, Z. Bai, C. Bischof, S. Blackford, J. Demmel, J. Dongarra, J. Du Croz, A. Greenbaum, S. Hammarling, A. McKenney, and D. Sorensen, LAPACK Users' Guide, 3rd ed. (Society for Industrial and Applied Mathematics, Philadelphia, PA, 1999). 
${ }^{74}$ B. Le Guennic, W. Hieringer, A. Görling, and J. Autschbach, J. Phys. Chem. A 109, $4836(2005)$.

${ }^{75}$ J. Kauczor and P. Norman, J. Chem. Theory Comput. 10, 2449 (2014).

${ }^{76}$ J. P. Perdew, K. Burke, and M. Ernzerhof, Phys. Rev. Lett. 77, 3865 (1996).

${ }^{77}$ J. P. Perdew, K. Burke, and M. Ernzerhof, Phys. Rev. Lett. 78, 1396 (1997).

${ }^{78}$ J. C. Slater, Phys. Rev. 81, 385 (1951).

${ }^{79}$ C. Adamo and V. Barone, J. Chem. Phys. 110, 6158 (1999).

${ }^{80}$ L. Visscher and K. G. Dyall, At. Data Nucl. Data Tables 67, 207 (1997).

${ }^{81}$ K. G. Dyall, Theor. Chem. Acc. 99, 366 (1998).

${ }^{82}$ K. G. Dyall, Theor. Chem. Acc. 115, 441 (2006).

${ }^{83}$ K. G. Dyall, Theor. Chem. Acc. 131, 1172 (2012).

${ }^{84}$ R. A. Kendall, T. H. Dunning Jr, and R. J. Harrison, J. Chem. Phys. 96, 6796 (1992).

${ }^{85}$ D. E. Woon and T. H. Dunning Jr, J. Chem. Phys. 98, 1358 (1993).

${ }^{86}$ A. K. Wilson, D. E. Woon, K. A. Peterson, and T. H. Dunning Jr, J. Chem. Phys. 110, 7667 (1999).

${ }^{87}$ K. Dyall and G. A.S.P., Unpublished, cVDZ basis sets for 3d elements.

${ }^{88}$ K. G. Dyall, Theor. Chem. Acc. 117, 483 (2007).

${ }^{89}$ K. G. Dyall, Theor. Chem. Acc. 112, 403 (2004).

${ }^{90}$ K. G. Dyall and A. S. Gomes, Theor. Chem. Acc. 125, 97 (2010).

${ }^{91}$ T. H. Dunning Jr, J. Chem. Phys. 90, 1007 (1989).

${ }^{92}$ E. Malkin, M. Repisky, S. Komorovsky, P. Mach, O. L. Malkina, and V. G. Malkin, J. Chem. Phys. 134, 044111 (2011).

${ }^{93}$ K. L. Bak, A. E. Hansen, K. Ruud, T. Helgaker, J. Olsen, and P. Jørgensen, Theor. Chim. Acta 90, 441 (1995).

${ }^{94}$ M. Pecul, K. Ruud, and T. Helgaker, Chem. Phys. Lett. 388, 110 (2004).

${ }^{95}$ J. Mattiat and S. Luber, Chem. Phys. 527, 110464 (2019).

${ }^{96}$ M. Rudolph and J. Autschbach, J. Phys. Chem. A 115, 2635 (2011).

${ }^{97}$ M. Rudolph and J. Autschbach, J. Phys. Chem. A 115, 14677 (2011). 

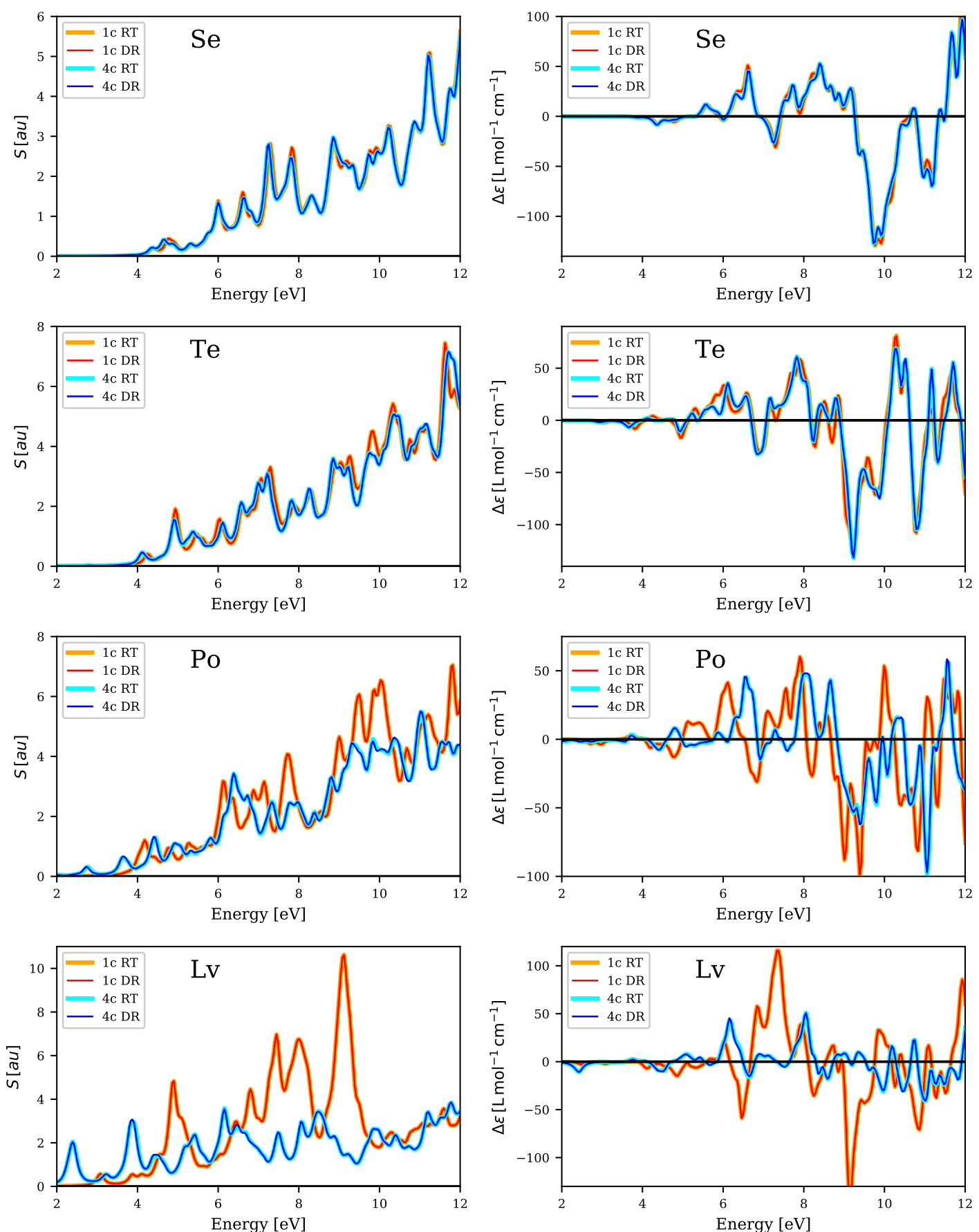

FIG. 1: Electronic absorption spectra (left) as the dipole strength function $S$ (Eq. (37)) and electronic circular dichroism spectra (right) as the differential extinction coefficient $\Delta \varepsilon$ (Eq. (45)) of dimethylchalcogeniranes $\mathrm{C}_{4} \mathrm{H}_{8} \mathrm{X}(\mathrm{X}=\mathrm{Se}, \mathrm{Te}, \mathrm{Po}, \mathrm{Lv})$ calculated at the $1 \mathrm{c}$ and 4c damped response (DR) and real-time (RT) TDDFT level of theory utilizing PBE functional. Spectra of lighter analogues $(\mathrm{X}=\mathrm{O}, \mathrm{S})$, as well $\mathrm{DR}$ results calculated with PBE0 functional, are available in the supplementary material. 

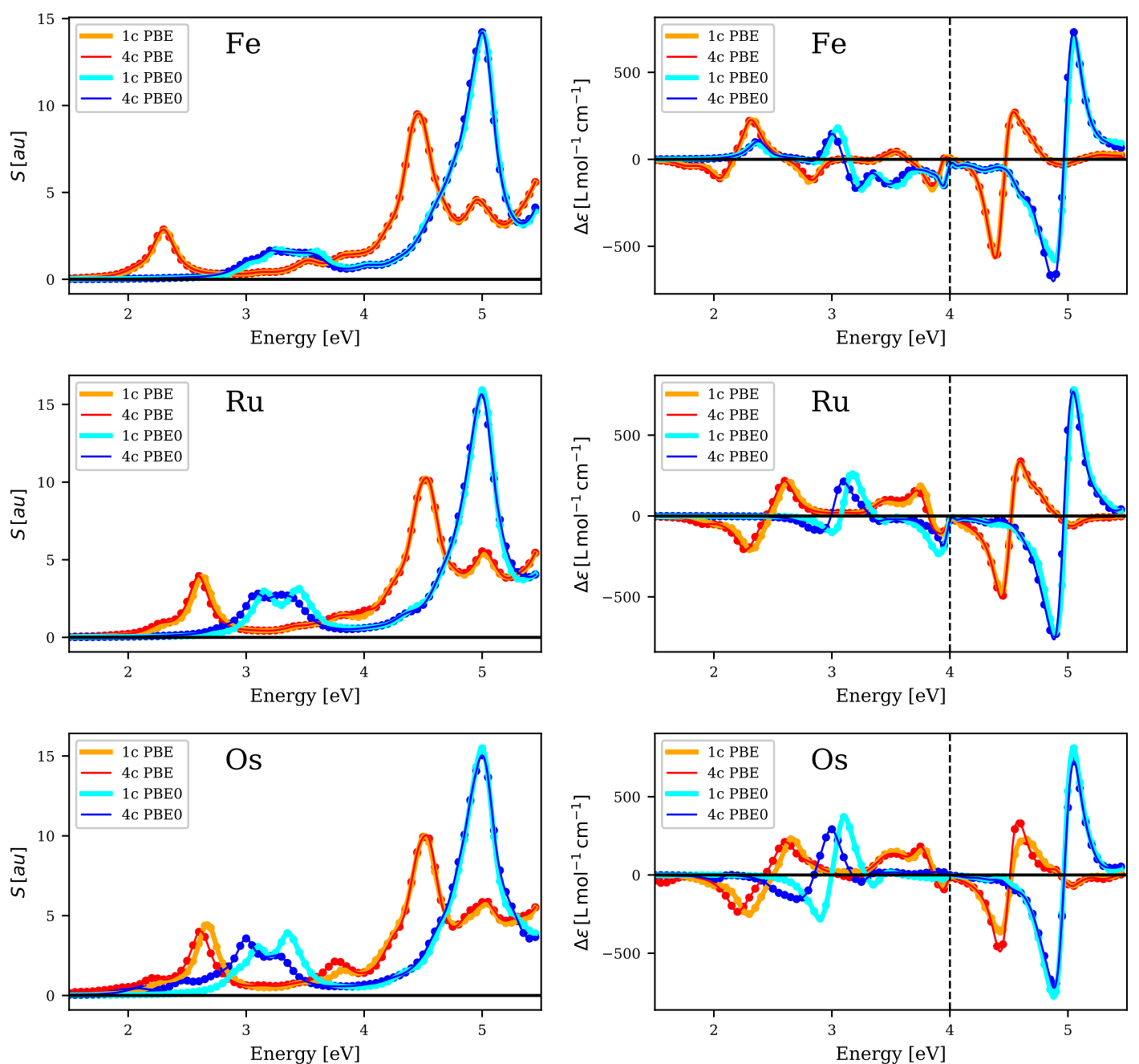

FIG. 2: Electronic absorption spectra (left) as the dipole strength function $S$ (Eq. (37)) and electronic circular dichroism spectra (right) as the differential extinction coefficient $\Delta \varepsilon$

(Eq. (45)) of $\Delta-\left[\mathrm{M}(\text { phen })_{3}\right]^{2+}(\mathrm{M}=\mathrm{Fe}, \mathrm{Ru}, \mathrm{Os})$ complexes calculated at the $1 \mathrm{c}$ and $4 \mathrm{c}$ linear damped response TDDFT level of theory utilizing PBE and PBE0 exchange-correlation functionals. Electronic circular dichroism spectra under $4 \mathrm{eV}$ were scaled by a factor of 6 to magnify the low-intensity transitions. 


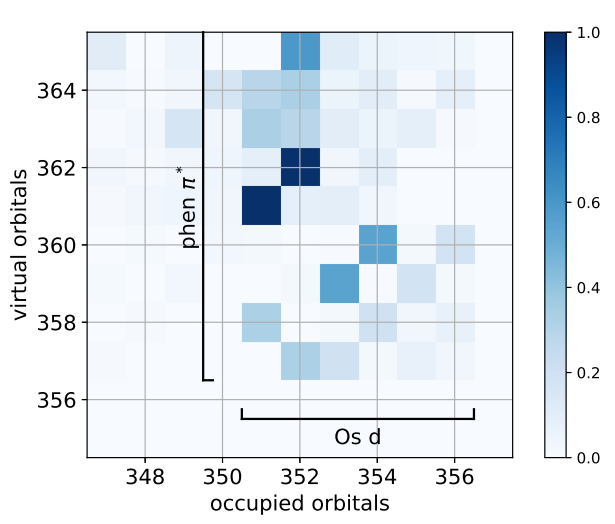

(a) ECD line at $2.6 \mathrm{eV}$

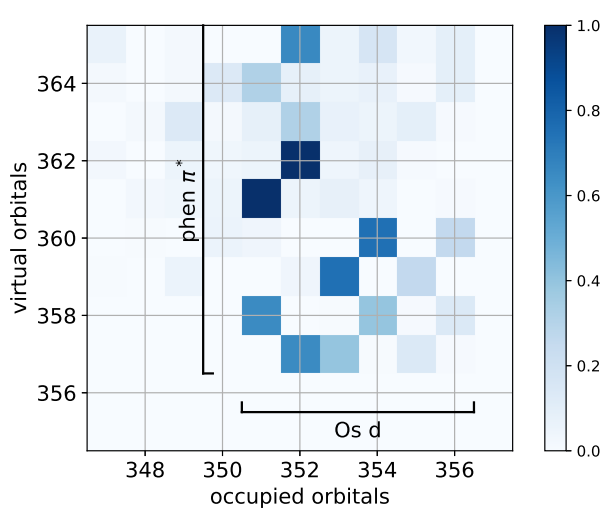

(c) EAS line at $2.6 \mathrm{eV}$

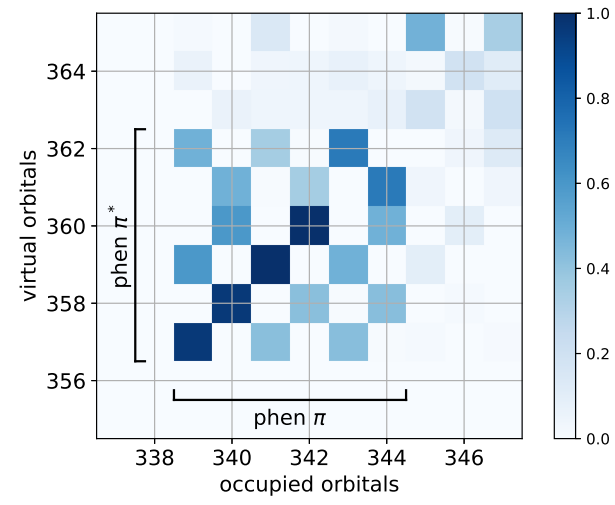

(b) ECD line at $4.5 \mathrm{eV}$

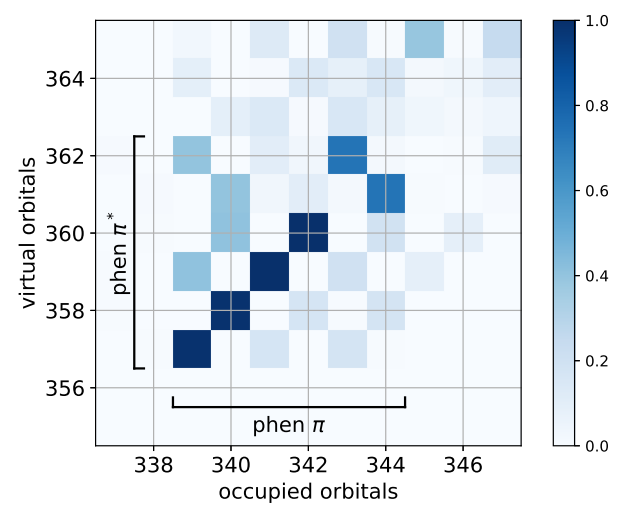

(d) EAS line at $4.5 \mathrm{eV}$

FIG. 3: Transition analysis of lines in electronic absorption (EAS) and electronic circular dichroism (ECD) spectra of $\left[\mathrm{Os}(\mathrm{phen})_{3}\right]^{2+}$ calculated at the PBE level of theory (see Fig. 2). The color intensity reflects the relative magnitude of the given occupied-virtual MO pair contribution. 

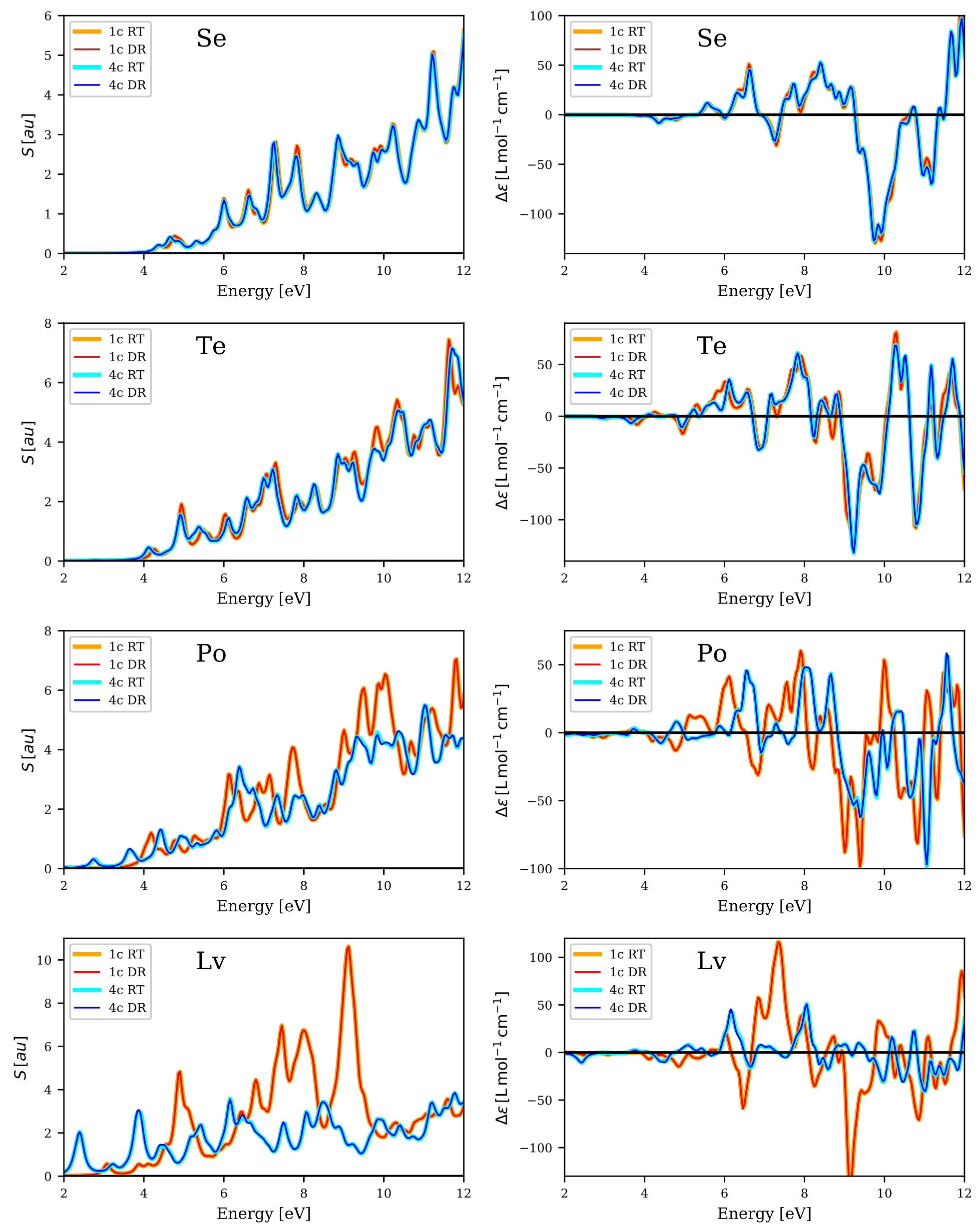

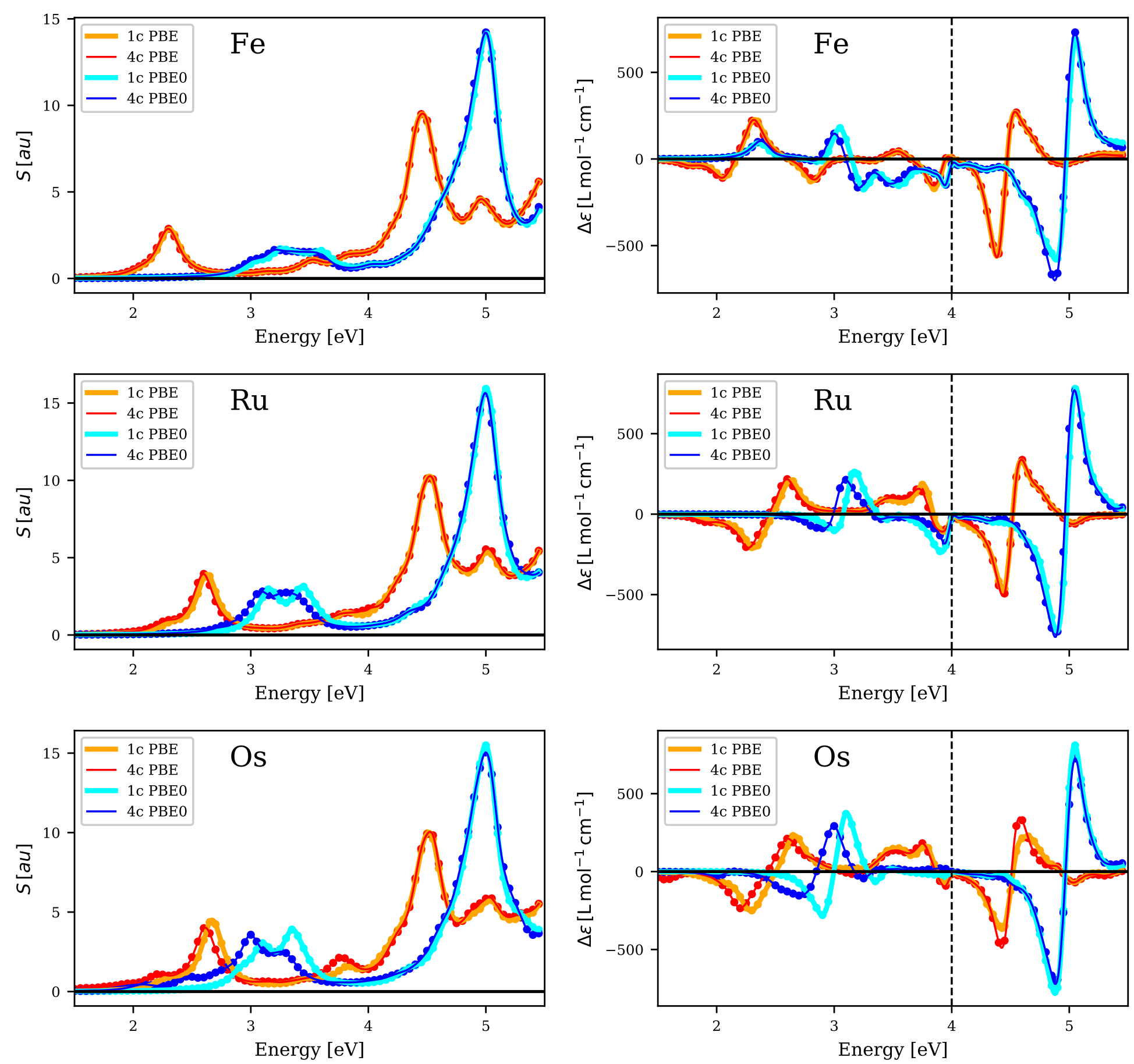


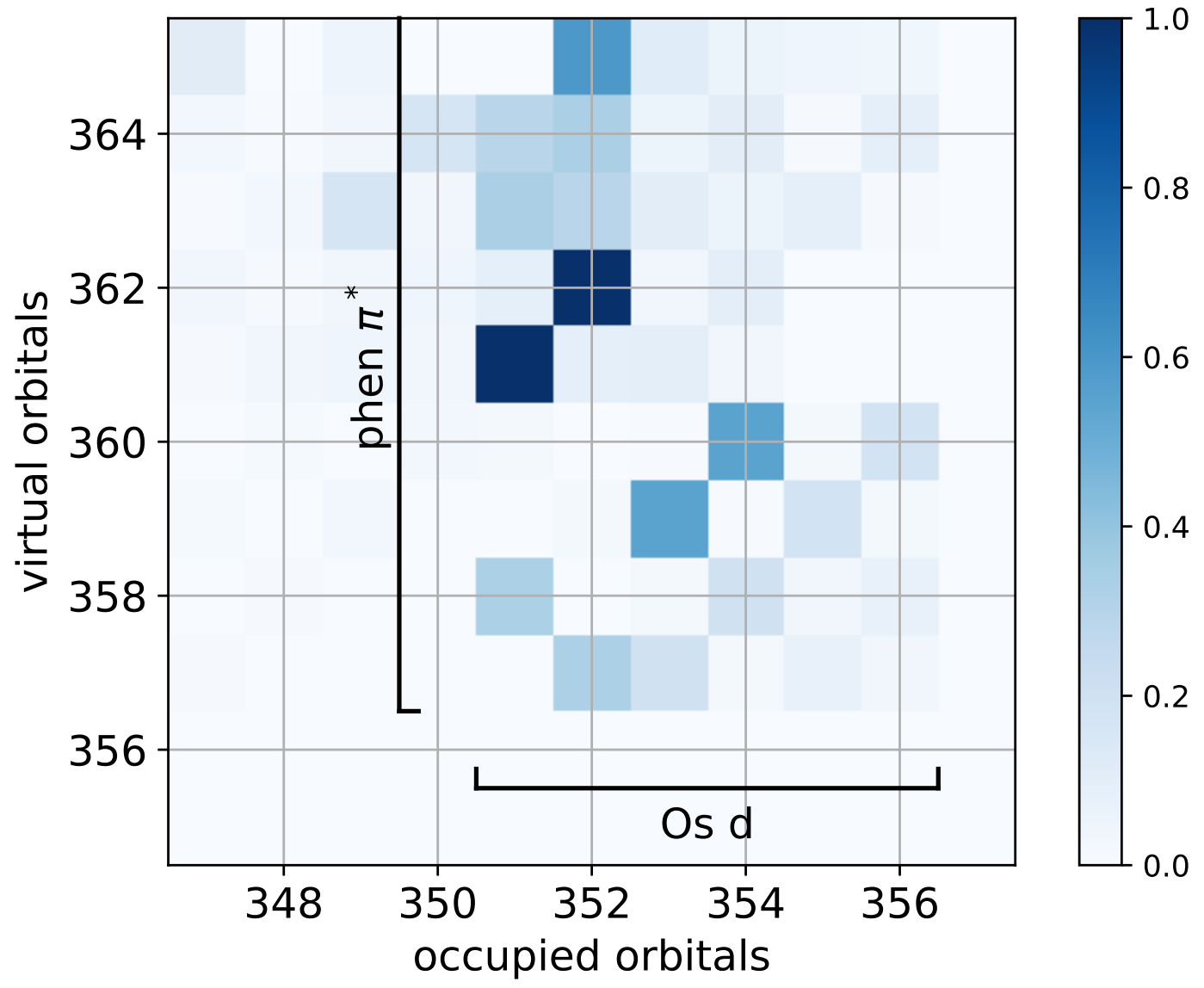




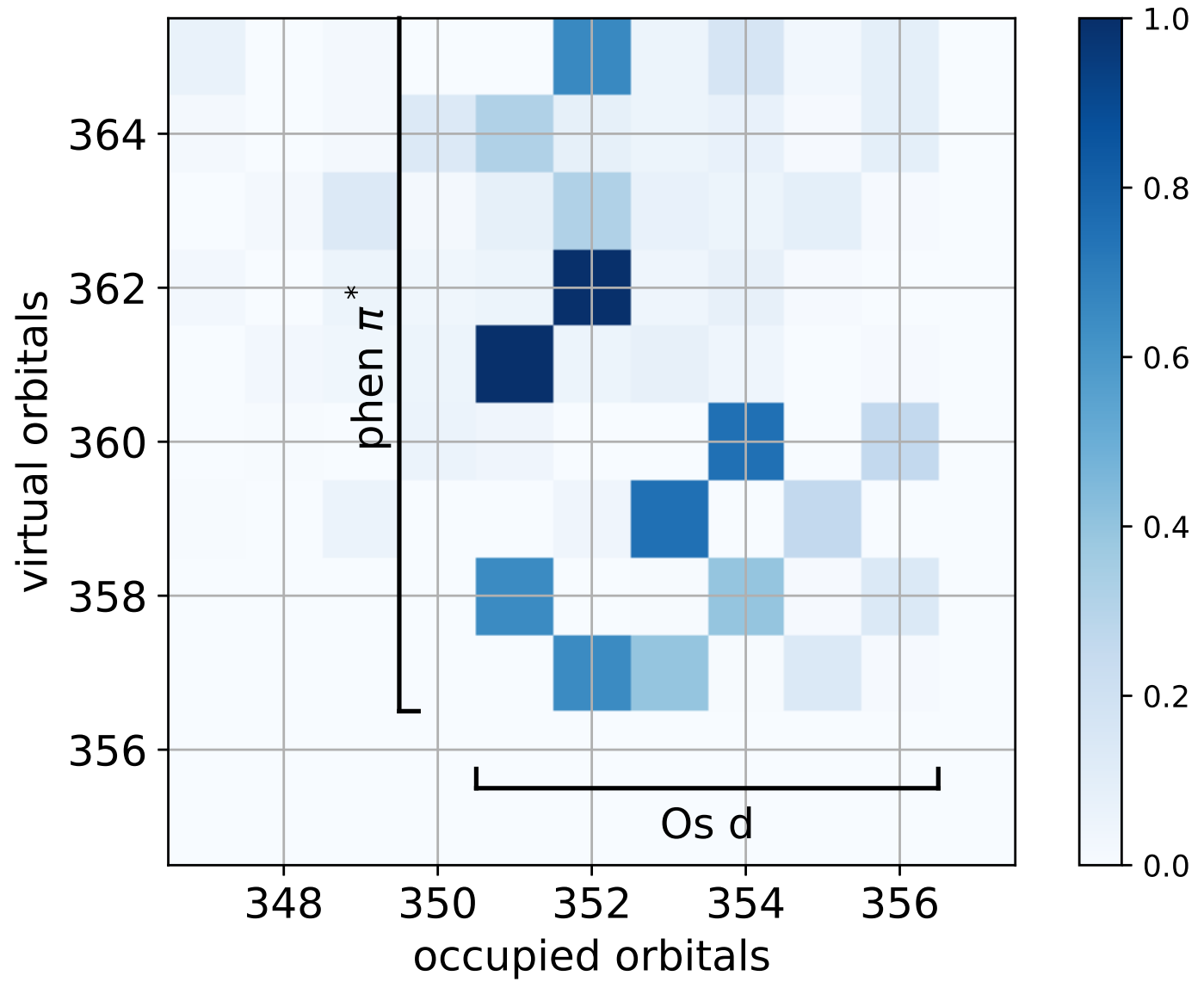




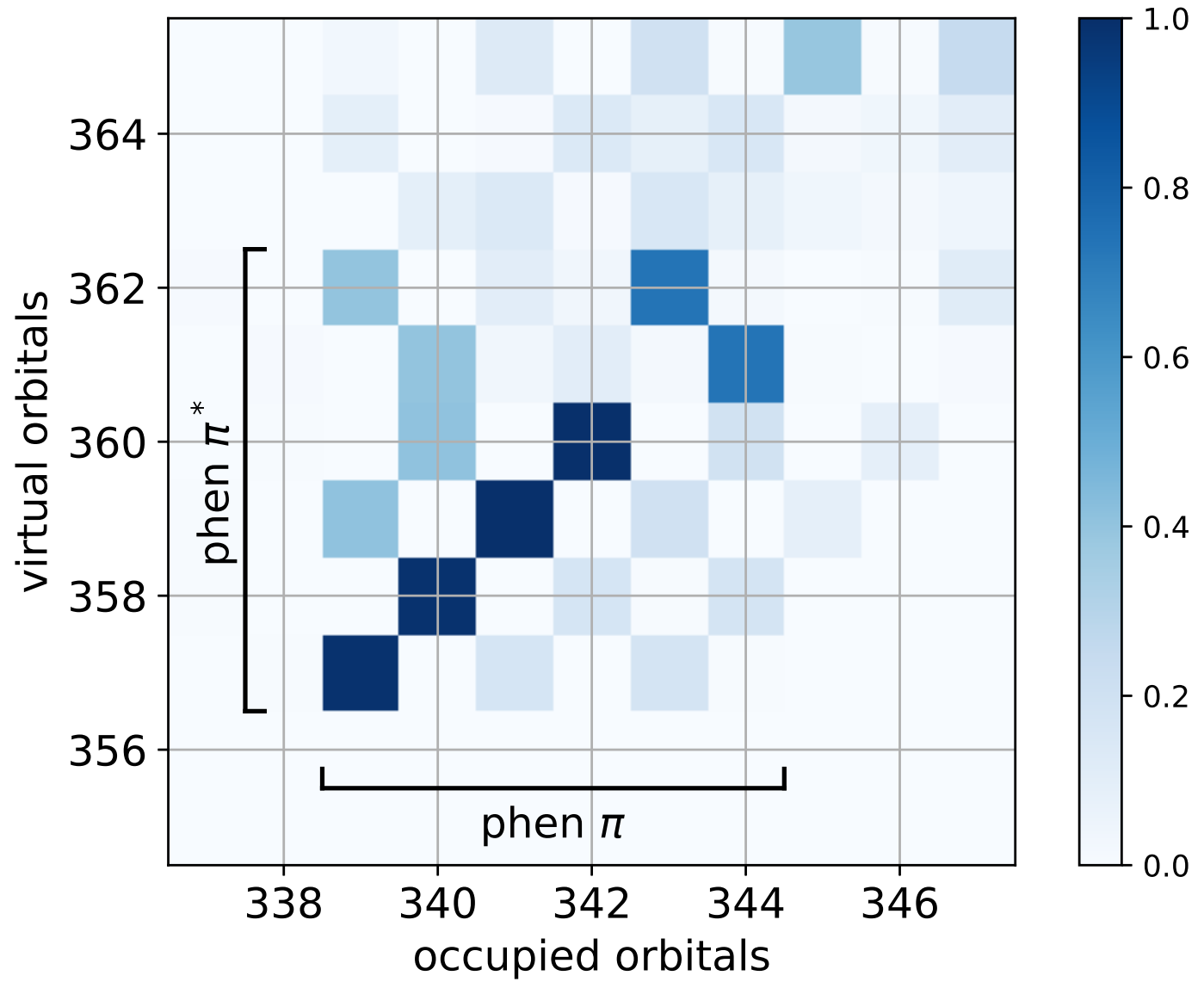

